\title{
Design of Distributed Fusion Predictor and Filter without Feedback for Nonlinear System with Correlated Noises and Random Parameter Matrices
}

\author{
Man-lu Liu ${ }^{1,2}$, Rui Lin ${ }^{2}$, Jian-wen Huo ${ }^{2, *}$, Li-guo Tan ${ }^{3, *}$, Qing Ling ${ }^{1}$, Eugene Yuryevich Zybin ${ }^{4}$ \\ ${ }^{1}$ School of Information Science and Technology, University of Science and Technology of China, Hefei 230026, China \\ ${ }^{2}$ School of Information Engineering, Southwest University of Science and Technology, Mianyang 621010, China \\ ${ }^{3}$ Research Center of Basic Space Science, Harbin Institute of Technology, Harbin 150001, China \\ ${ }^{4}$ State Research Institute of Aviation Systems (GosNIIAS), Moscow 125319, Russia \\ *Corresponding author: huojianwen2008@hotmail.com,tanliguo@hit.edu.cn
}

\begin{abstract}
This work presents distributed predictor and filter without feedback for nonlinear stochastic uncertain system with correlated noises. Firstly, for the problem that the process noise and measurement noise are correlated, the two-step prediction theorem based on projection theorem is used to replace the one-step prediction theorem, and the two-step prediction value of a single sensor is obtained. Secondly, the two-step prediction value of each sensor state is used as the measurement information to modify the distributed fusion predictor to obtain the distributed fusion prediction value. Then, according to the projection theorem, the prediction value of distributed fusion is used as measurement information to modify the filtering value of distributed fusion. Finally, the Cubature Kalman filter (CKF) algorithm is used to implement the algorithm proposed in this paper. By comparison with existing methods, the algorithm proposed in this paper solves the problem that existing methods cannot handle state estimation and prediction problems for nonlinear multi-sensor stochastic uncertain systems with correlated noises.
\end{abstract}

Keywords: nonlinear stochastic uncertain system, distributed fusion, state estimation.

\section{INTRODUCTION}

In recent years, multi-sensor systems have been widely used in target tracking and positioning [1]-[2], intelligent perception [3]-[4], and pattern recognition [5]-[6] because multi-sensor systems can enhance the robustness of the system and improve the estimation accuracy of the system and accompanied by a large number of data processing algorithms. Multi-sensor data processing algorithms can be roughly divided into centralized, distributed, and sequential. Considering that distributed fusion has the advantages of flexible structure, easy fault isolation, and small amount of calculation, it has received widespread attention.

According to different fusion criteria, various distributed fusion algorithms have been formed. Literature [7]-[8] designed a federated filter based on information distribution criteria, which also has the advantages of distributed and centralized fusion. Literature [9] gives the structure of the federated filter including the main filter. However, the federated filter involves weight distribution problems. Literature [10]-[12] carried out weighted fusion algorithm design according to three different criteria, weighted by matrix, weighted by diagonal matrix, and weighted by scalar. However, the above algorithm needs to calculate the crosscovariance matrix, which puts forward higher requirements on the prior information of the system. Therefore, literature [13] give a new covariance cross fusion algorithm that does not need to calculate the cross-covariance information. However, the weight calculation of the covariance intersection fusion algorithm needs to solve the multidimensional optimization problem. Literature [14] designed a kind of sequential covariance intersection fusion algorithm which transforms the multi-dimensional optimization problem into multiple one-dimensional optimization problems, which can be solved by the golden section method or the Fibonacci method [15]. In order to eliminate the influence of the fusion sequence on the fusion result and reduce the amount of calculation, literature [16] proposed a sequence-insensitive covariance intersection-fusion algorithm, and introduced two fusion methods, namely batch fusion and sequential fusion. In the past two years, literature [17] provided a novel data fusion algorithm, which is different from the previous form of combining and weighting the corrected measurements of local sensors. Instead, it 
regards the local estimation as measurement information which is used to modify one-step prediction information. Modifications, from a formal point of view, are more towards centralized fusion algorithm processing ideas. Literature [18] further studied the design of the optimal predictor and filter with random parameter matrix and related noise for this method. However, it did not consider the nonlinear situation.

Considering that most of the actual systems are nonlinear systems, this paper is inspired by literature [18], based on the minimum mean square error criterion, the distributed fusion Gaussian predictor and filter design with random parameter matrix and related noise are studied. The rest of the paper is organized as follows: Section 2 describes the problem to be studied, Section 3 introduces the design of the distributed fusion predictor and filter, Section 4 gives the numerical implementation of the algorithm, simulation verification is carried out in Section 5, and the conclusion of this paper is given in the last section.

\section{PROBLEM FORMULATION}

Considering the following discrete time nonlinear multisensor stochastic uncertain systems:

$$
\begin{gathered}
x_{k+1}=\widehat{\Phi}_{k} f\left(x_{k}\right)+\widehat{\Gamma}_{k} \omega_{k} \\
y_{k}^{i}=\hat{H}_{k}^{i} h^{i}\left(x_{k}\right)+\widehat{D}_{k}^{i} v_{k}^{i}, i=1, \cdots, N
\end{gathered}
$$

where $x_{\mathrm{k}} \in \mathrm{R}^{\mathrm{n}}$ is the system state; $y_{k}^{i} \in R^{m_{i}}, i=1, \cdots, N$ is the measurement of multi-sensors; $\omega_{k} \in R^{r}$ is the system noise; $\quad v_{k}^{i} \in R^{s_{i}}, i=1, \cdots, N \quad$ is the sensor noise; $\widehat{\Phi}_{\mathrm{k}}, \widehat{\Gamma}_{\mathrm{k}}, \hat{H}_{k}^{i}, \widehat{D}_{k}^{i}$ are the correlated stochastic parameter matrices with suitable dimensions at $k$ epoch; $N$ is the number of sensors; Superscript $i$ represents the $i$ th sensor.

Assumption 1: Stochastic parameter matrices $\widehat{\Phi}_{k}, \widehat{\Gamma}_{k}$, $\hat{H}_{k}^{i}$ and $\hat{D}_{k}^{i}, i=1, \cdots N$ are correlated with each other at the same epoch and are independent of system noise $\omega_{k}$ and $v_{k}^{i}, i=1, \cdots N$. They satisfy the following statistical properties:

$$
\begin{aligned}
& E\left[\widehat{\Phi}_{k}\right]=\Phi_{k}, E\left[\widehat{\Gamma}_{k}\right]=\Gamma_{k}, E\left[\hat{H}_{k}^{i}\right]=H_{k}^{i} \\
& E\left[\widehat{D}_{k}^{i}\right]=D_{k}^{i}, E\left[\widehat{\mathrm{M}}_{k}^{i j} \widehat{G}_{l}^{s u}\right]=T_{k l}^{\hat{M}^{i j} \widehat{G}^{s u}} \delta_{k l} \\
& \widehat{\mathrm{M}}, \widehat{G}=\widehat{\Phi}, \widehat{\Gamma}, \hat{H}^{i}, \widehat{D}^{i}
\end{aligned}
$$

where $\widehat{M}_{k}^{i j}$ and $\widehat{G}_{l}^{s u}$ represent the element at $(i, j)$ and $(s, u)$ in $\widehat{M}_{k}$ and $\widehat{G}_{l}$, respectively.
Assumption 2: $\omega_{k}$ and $v_{k}^{i}, i=1, \cdots, N$ are correlated white noise and satisfied with

$$
E\left\{\left[\begin{array}{c}
\omega_{k} \\
v_{k}^{i}
\end{array}\right]\left[\begin{array}{ll}
\omega_{l}^{T} & \left(v_{l}^{j}\right)^{T}
\end{array}\right]\right\}=\left[\begin{array}{cc}
Q_{k} & S_{k}^{j} \\
\left(S_{k}^{i}\right)^{T} & R_{k}^{i j}
\end{array}\right] \delta_{k l}
$$

Define $\quad \tilde{M}_{k}=\widehat{M}_{k}-M_{k}, M=\Phi, \Gamma, H, D \quad$, then $E[\tilde{M}]=0$. Define $E\left[\tilde{M}_{k} \theta_{k} \eta_{k}^{T} \tilde{G}_{k}^{T}\right]=\Omega_{k}^{\tilde{M} \tilde{G}}\left(q_{k}^{\theta \eta}\right)$, where, $\theta_{k}$ and $\eta_{k}$ are independent with $\widehat{M}_{k}$ and $\widehat{G}_{k}$, and we have $q_{k}^{\theta \eta}=E\left[\theta_{k} \eta_{k}^{T}\right]$.

Notes: Derivating the matrix trace yields

$$
\begin{aligned}
& \frac{\partial \operatorname{tr}(\bar{A} \bar{B})}{\partial A}=\frac{\partial \operatorname{tr}(\bar{B} \bar{A})}{\partial \bar{A}}=\bar{B}^{T} \\
& \frac{\partial \operatorname{tr}\left(\bar{A}^{T} \bar{B}\right)}{\partial \bar{A}}=\frac{\partial \operatorname{tr}\left(\bar{B} \bar{A}^{T}\right)}{\partial \bar{A}}=\bar{B} \\
& \frac{\partial \operatorname{tr}\left(\bar{A}^{T} \bar{B} \bar{A}\right)}{\partial \bar{A}}=\bar{B} \bar{A}+\bar{B}^{T} \bar{A} \\
& \frac{\partial \operatorname{tr}\left(\bar{A} \bar{B} \bar{A}^{T}\right)}{\partial \bar{A}}=\bar{A} \bar{B}+\bar{A} \bar{B}^{T}
\end{aligned}
$$

The system and sensor noises are refactored as $W_{k}=\tilde{\Gamma}_{k} \omega_{k}+\Gamma_{k} \omega_{k}, \quad i=1, \cdots, N$, and (1) and (2) are rewritten as

$$
\begin{gathered}
x_{k+1}=\Phi_{k} f\left(x_{k}\right)+\tilde{\Phi}_{k} f\left(x_{k}\right)+W_{k} \\
y_{k}^{i}=H_{k}^{i} h^{i}\left(x_{k}\right)+\tilde{H}_{k}^{i} h^{i}\left(x_{k}\right)+V_{k}^{i}, i=1, \cdots, N
\end{gathered}
$$

The refactored noises are satisfied as:

$$
\begin{aligned}
& E\left[W_{k}\right]=0, E\left[V_{k}^{i}\right]=0 \\
& Q_{k}^{W W}=E\left[W_{k} W_{k}^{T}\right]=\Omega_{k}^{\tilde{\Gamma} \tilde{\Gamma}} Q_{k}+\Gamma_{k} Q_{k} \Gamma_{k}^{T} \\
& R_{k}^{V^{i} V^{j}}=E\left[V_{k}^{i}\left(V_{k}^{j}\right)^{T}\right]=\Omega_{k}^{\tilde{D}^{i} \tilde{D}^{j}} R_{k}^{i j}+D_{k}^{i} R_{k}^{i j}\left(D_{k}^{j}\right)^{T} \\
& S_{k}^{W V^{i}}=E\left[W_{k}\left(V_{k}^{i}\right)^{T}\right]=\Omega_{k}^{\tilde{\Gamma} \tilde{D}^{i}} S_{k}^{i}+\Gamma_{k} S_{k}^{i}\left(D_{k}^{i}\right)^{T}
\end{aligned}
$$

\section{DESIGN OF DISTRIBUTED FUSION PREDICTOR AND FILTER}

The flowchart of the proposed algorithm for a two-sensor scenario is shown as follows: 


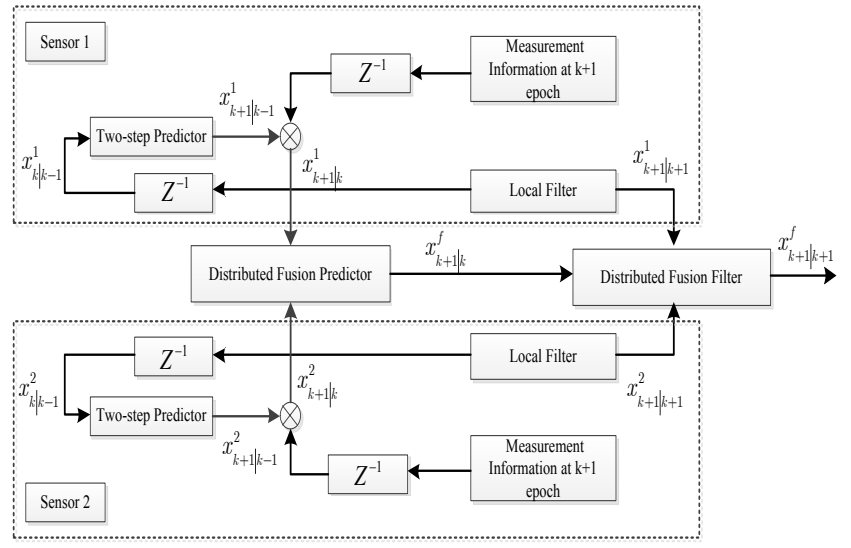

Fig.1. The data processing flowchart for two-sensor scenario.

Lemma 1: The local predictor is designed as

$$
\begin{gathered}
x_{k+1 \mid k}^{i}=x_{k+1 \mid k-1}^{i}+K_{k+1, k \mid k-1}^{x^{i} y^{i}} e_{k}^{i} \\
K_{k+1, k \mid k-1}^{x^{i} y^{i}}=P_{k+1, k \mid k-1}^{x^{i} y^{i}}\left(Q_{k}^{e^{i}}\right)^{-1}
\end{gathered}
$$

where,

$$
\begin{aligned}
x_{k+1 \mid k-1}^{i} & =E\left[x_{k+1} \mid Y_{k-1}^{i}\right] \\
& =E\left[\Phi_{k} f\left(x_{k}\right)+\tilde{\Phi}_{k} f\left(x_{k}\right)+W_{k} \mid Y_{k-1}^{i}\right] \\
& =\Phi_{k} \int f\left(x_{k}\right) N_{1}^{k} d x_{k}
\end{aligned}
$$

where, $Y_{k-1}^{i}=L\left\{y_{1}^{i}, \cdots, y_{k-1}^{i}\right\}$ represents the space formed by the elements in $L$ and $N_{1}^{k}=N\left(x_{k} ; x_{k \mid k-1}^{i}, P_{k \mid k-1}^{i}\right)$.

$$
\begin{aligned}
& P_{k+1, k \mid k-1}^{x^{i} y^{i}}=E\left[\left(x_{k+1}-x_{k+1 \mid k-1}^{i}\right)\left(y_{k}^{i}-y_{k \mid k-1}^{i}\right)^{T} \mid Y_{k-1}^{i}\right] \\
& =E\left[\begin{array}{l}
\left(\Phi_{k} \tilde{f}\left(x_{k \mid k-1}^{i}\right)+\tilde{\Phi}_{k} f\left(x_{k}\right)+W_{k}\right) \\
\left(H_{k}^{i} \tilde{h}^{i}\left(x_{k \mid k-1}^{i}\right)+\tilde{H}_{k}^{i} h^{i}\left(x_{k}\right)+V_{k}^{i}\right)^{T} \mid Y_{k-1}^{i}
\end{array}\right] \\
& =\Phi_{k} E\left[\tilde{f}\left(x_{k \mid k-1}^{i}\right)\left(\tilde{h}^{i}\left(x_{k \mid k-1}^{i}\right)\right)^{T} \mid Y_{k-1}^{i}\right]\left(H_{k}^{i}\right)^{T} \\
& +\Omega_{k}^{\tilde{\Phi} \tilde{H}^{i}} E\left[f\left(x_{k}\right)\left(h^{i}\left(x_{k}\right)\right)^{T} \mid Y_{k-1}^{i}\right]+S_{k}^{W V^{i}} \\
& =\Phi_{k}\left(\int \tilde{f}\left(x_{k \mid k-1}^{i}\right)\left(\tilde{h}^{i}\left(x_{k \mid k-1}^{i}\right)\right)^{T} N_{1}^{k} d x_{k}\right)\left(H_{k}^{i}\right)^{T} \\
& +\Omega_{k}^{\tilde{\Phi} \tilde{H}^{i}} \int f\left(x_{k}\right)\left(h^{i}\left(x_{k}\right)\right)^{T} N_{1}^{k} d x_{k}+S_{k}^{W V^{i}}
\end{aligned}
$$

where, $\quad \tilde{f}\left(x_{k \mid k-1}^{i}\right)=f\left(x_{k}\right)-f\left(x_{k \mid k-1}^{i}\right)$ $\tilde{h}^{i}\left(x_{k \mid k-1}^{i}\right)=h^{i}\left(x_{k}\right)-h^{i}\left(x_{k \mid k-1}^{i}\right)$. According to the definition of error, we have

$$
\begin{aligned}
x_{k+1}-x_{k+1 \mid k}^{i} & =\Phi_{k} f\left(x_{k}\right)+\tilde{\Phi}_{k} f\left(x_{k}\right) \\
& +W_{k}-\Phi_{k} f\left(x_{k \mid k-1}^{i}\right)-K_{k+1, k \mid k-1}^{x^{i} y^{i}} e_{k}^{i} \\
= & \Phi_{k} \tilde{f}\left(x_{k \mid k-1}^{i}\right)+\tilde{\Phi}_{k} f\left(x_{k}\right)+W_{k} \\
& -K_{k+1, k \mid k-1}^{x^{i} y^{i}}\left(H_{k}^{i} \tilde{h}^{i}\left(x_{k \mid k-1}^{i}\right)+\tilde{H}_{k}^{i} h^{i}\left(x_{k}\right)+V_{k}^{i}\right) \\
= & \left(\Phi_{k} \tilde{f}\left(x_{k \mid k-1}^{i}\right)-K_{k+1, k \mid k-1}^{x^{i} y^{i}} H_{k}^{i} \tilde{h}^{i}\left(x_{k \mid k-1}^{i}\right)\right) \\
& +\left(\tilde{\Phi}_{k} f\left(x_{k}\right)-K_{k+1, k \mid k-1}^{x^{i} y^{i}} \tilde{H}_{k}^{i} h^{i}\left(x_{k}\right)\right) \\
& +\left(W_{k}-K_{k+1, k \mid k-1}^{x^{i} y^{i}} V_{k}^{i}\right)
\end{aligned}
$$

Considering that the three items in the brackets in (12) are not correlated to each other, there are

$$
\begin{aligned}
P_{k+1 \mid k}^{x^{i} x^{j}} & =E\left[\left(x_{k+1}-x_{k+1 \mid k}^{i}\right)\left(x_{k+1}-x_{k+1 \mid k}^{j}\right)^{T} \mid Y_{k}^{i}, Y_{k}^{j}\right] \\
& =\Psi_{1}+\Psi_{2}+\Psi_{3}
\end{aligned}
$$

where,

$$
\begin{aligned}
& \Psi_{1}=E\left[\begin{array}{l}
\left(\Phi_{k} \tilde{f}\left(x_{k \mid k-1}^{i}\right)-K_{k+1, k \mid k-1}^{x^{i} y^{i}} H_{k}^{i} \tilde{h}^{i}\left(x_{k \mid k-1}^{i}\right)\right) \\
\left(\Phi_{k} \tilde{f}\left(x_{k \mid k-1}^{j}\right)-K_{k+1, k \mid k-1}^{x^{j} y^{j}} H_{k}^{j} \tilde{h}^{j}\left(x_{k \mid k-1}^{j}\right)\right)^{T}
\end{array}\right] \\
& =\Phi_{k} E\left[\tilde{f}\left(x_{k \mid k-1}^{i}\right) \tilde{f}^{T}\left(x_{k \mid k-1}^{j}\right)\right] \Phi_{k}^{T} \\
& +K_{k+1, k \mid k-1}^{x^{i} y^{i}} H_{k}^{i} E\left[\tilde{h}^{i}\left(x_{k \mid k-1}^{i}\right)\left(\tilde{h}^{j}\left(x_{k \mid k-1}^{j}\right)\right)^{T}\right]\left(K_{k+1, k \mid k-1}^{x^{j} y^{j}} H_{k}^{j}\right)^{T} \\
& -K_{k+1, k \mid k-1}^{x^{i} y^{i}} H_{k}^{i} E\left[\tilde{h}^{i}\left(x_{k \mid k-1}^{i}\right)\left(\tilde{f}\left(x_{k \mid k-1}^{j}\right)\right)^{T}\right] \Phi_{k}^{T} \\
& -\Phi_{k} E\left[\tilde{f}\left(x_{k \mid k-1}^{i}\right)\left(\tilde{h}^{j}\left(x_{k \mid k-1}^{j}\right)\right)^{T}\right]\left(K_{k+1, k \mid k-1}^{x^{j} y^{j}} H_{k}^{j}\right)^{T}
\end{aligned}
$$




$$
\begin{aligned}
& \Psi_{2}=E\left[\begin{array}{l}
\left(\tilde{\Phi}_{k} f\left(x_{k}\right)-K_{k+1, k \mid k-1}^{x^{i} y^{i}} \tilde{H}_{k}^{i} h^{i}\left(x_{k}\right)\right) \\
\left.\left(\tilde{\Phi}_{k} f\left(x_{k}\right)-K_{k+1, k \mid k-1}^{x^{j} y^{j}} \tilde{H}_{k}^{i} h^{j}\left(x_{k}\right)\right)^{T}\right]
\end{array}\right. \\
& =\Omega_{k}^{\tilde{\Phi} \tilde{\Phi}} E\left[f\left(x_{k}\right) f^{T}\left(x_{k}\right)\right] \\
& -K_{k+1, k \mid k-1}^{x^{i} y^{i}} \Omega_{k}^{\tilde{H}^{i} \tilde{\Phi}_{k}} E\left[h^{i}\left(x_{k}\right) f^{T}\left(x_{k}\right)\right] \\
& -\Omega_{k}^{\tilde{\Phi}_{k} \tilde{H}^{j}}\left[E\left[f\left(x_{k}\right)\left(h^{j}\left(x_{k}\right)\right)^{T}\right]\right)\left(K_{k+1, k \mid k-1}^{x^{j} y^{j}}\right)^{T} \\
& +K_{k+1, k \mid k-1}^{x^{i} y^{i}} \Omega_{k}^{\tilde{H}^{i} \tilde{H}^{j}} E\left[h^{i}\left(x_{k}\right)\left(h^{j}\left(x_{k}\right)\right)^{T}\right]\left(K_{k+1, k \mid k-1}^{x^{j} y^{j}}\right)^{T}
\end{aligned}
$$$$
\begin{aligned}
& \Psi_{3}=E\left[\begin{array}{l}
\left(W_{k}-K_{k+1, k \mid k-1}^{x^{i} y^{i}} V_{k}^{i}\right) \\
\left(W_{k}-K_{k+1, k \mid k-1}^{x^{j} y^{j}} V_{k}^{j}\right)^{T}
\end{array}\right] \\
& =Q_{k}^{W W}-S_{k}^{W V^{j}}\left(K_{k+1, k \mid k-1}^{x^{j} y^{j}}\right)^{T} \\
& +K_{k+1, k \mid k-1}^{x^{i} y^{i}} R_{k}^{V^{i} V^{j}}\left(K_{k+1, k \mid k-1}^{x^{j} y^{j}}\right)^{T} \\
& -K_{k+1, k \mid k-1}^{x^{i} y^{i}}\left(S_{k}^{W V^{i}}\right)^{T}
\end{aligned}
$$

where,

$$
\begin{aligned}
& E\left[\tilde{f}\left(x_{k \mid k-1}^{i}\right) \tilde{f}^{T}\left(x_{k \mid k-1}^{j}\right) \mid Y_{k-1}^{i}, Y_{k-1}^{j}\right. \\
& =\int \tilde{f}\left(x_{k \mid k-1}^{i}\right) \tilde{f}^{T}\left(x_{k \mid k-1}^{j}\right) N_{2}^{k} d\left[\begin{array}{l}
x_{k} \\
x_{k}
\end{array}\right]
\end{aligned}
$$

where,

$$
N_{2}^{k}=N\left(\left[\begin{array}{c}
x_{k} \\
x_{k}
\end{array}\right] ;\left[\begin{array}{c}
x_{k \mid k-1}^{i} \\
x_{k \mid k-1}^{j}
\end{array}\right],\left[\begin{array}{cc}
P_{k \mid k-1}^{x^{i} x^{i}} & P_{k \mid k-1}^{x^{i} x^{j}} \\
\left(P_{k \mid k-1}^{x^{i} x^{j}}\right)^{T} & P_{k \mid k-1}^{x^{j} x^{j}}
\end{array}\right]\right)
$$

$E\left[g_{1}\left(g_{2}\right)^{T} \mid \eta_{1}, \eta_{2}\right]$ representing $g_{1}$ is on the condition

$\eta_{1}$, and $g_{2}$ is on the condition $\eta_{2}, g_{1}$ and $g_{2}$ represent nonlinear functions, $\eta_{1}$ and $\eta_{2}$ represent the obtained measurement. For the $i$ th sensor, the initial values are taken as $x_{0 \mid-1}^{i}=\mu_{0}, P_{0 \mid-1}^{x^{i} x^{j}}=P_{0}$.

The other expected terms in equations (14) - (16) are the same as the linear space of formula (17), so the probability density function will not be repeated here.
Taking $\Psi_{1}, \Psi_{2}, \Psi_{3}$ into (13) yields the one-step prediction covariance. Then, taking (10), (11) into (8), (9) yields the mean of the one-step prediction.

Next, using the local one-step prediction to modify the prior information of the fusion center, let $X_{k+1}=e x_{k+1}$. Then, we can get the one-step prediction $X_{k+1 \mid k}=\left[x_{k+1 \mid k}^{1}, \cdots, x_{k+1 k}^{N}\right]^{T}$ and the one-step prediction error $\quad \tilde{X}_{k+1 \mid k}=\left[x_{k+1}-x_{k+1 \mid k}^{1}, \cdots, x_{k+1}-x_{k+1 \mid k}^{N}\right]^{T} \quad$ of $X_{k+1}$,

Theorem 1: For the system (5) and (6), based on the assumption 1 and 2 , the distributed optimal fusion predictor is designed as follows:

$$
\begin{aligned}
& x_{k+1 \mid k}^{f}= x_{k+1 \mid k-1}^{f}+L_{k+1}\left(\hat{X}_{k+1 \mid k}-e x_{k+1 \mid k-1}^{f}\right) \\
&=\left(I_{n}-L_{k+1} e\right) \Phi_{k} f\left(x_{k \mid k-1}^{f}\right)+L_{k+1} \hat{X}_{k+1 \mid k} \\
& P_{k+1 \mid k}^{f}=E\left[\tilde{x}_{k+1 \mid k}^{f}\left(\tilde{x}_{k+1 \mid k}^{f}\right)^{T} \mid Y_{k}\right] \\
&=\left(I_{n}-L_{k+1} e\right)\left\{\begin{array}{l}
\Phi_{k} E\left[\tilde{f}\left(x_{k \mid k-1}^{f}\right) \tilde{f}^{T}\left(x_{k \mid k-1}^{f}\right)\right] \Phi_{k}^{T} \\
+\Omega_{k}^{\tilde{\Phi} \tilde{\Phi}} E\left[f\left(x_{k}\right) f^{T}\left(x_{k}\right)\right]+Q_{k}^{W W}
\end{array}\right\} \\
& \times\left(I_{n}-L_{k+1} e\right)^{T}+L_{k+1} \Xi_{k+1 \mid k} L_{k+1}^{T}+\Lambda+\Lambda^{T} \\
& L_{k+1}=\left(\begin{array}{l}
\left(A+B+Q_{k}^{W W} e^{T}-D\right) \\
-2\left\{C+Q_{k}^{W W}\right\} e^{T}
\end{array}\right)^{-1} \\
& \times\left(\begin{array}{l}
e A+(e A)^{T}+B e+(B e)^{T} \\
+\left(e Q_{k}^{W W} e^{T}-e D-(e D)^{T}\right) \\
-e C e^{T}-\Xi_{k+1 \mid k}
\end{array}\right.
\end{aligned}
$$

The proof process of the above three formulas is shown in Appendix 1.

Lemma 2: For systems (5) and (6), innovation and its covariance are calculated as follows:

$$
e_{k+1}^{i}=y_{k+1}^{i}-y_{k+1 \mid k}^{i}
$$

where, 


$$
\begin{aligned}
& y_{k+1 \mid k}^{i}=E\left[y_{k+1}^{i} \mid Y_{k}\right] \\
& =E\left[H_{k+1}^{i} h^{i}\left(x_{k+1}\right)+\tilde{H}_{k+1}^{i} h^{i}\left(x_{k+1}\right)+V_{k+1}^{i} \mid Y_{k}^{i}\right] \\
& =H_{k+1}^{i} \int h^{i}\left(x_{k+1}\right) N_{1}^{k+1} d x_{k+1}
\end{aligned}
$$

For simplicity, rewrite $e_{k+1}^{i}$ as

$$
\begin{aligned}
& e_{k+1}^{i}=y_{k+1}^{i}-y_{k+1 \mid k}^{i} \\
& =H_{k+1}^{i} h^{i}\left(x_{k+1}\right)+\tilde{H}_{k+1}^{i} h^{i}\left(x_{k+1}\right) \\
& +V_{k+1}^{i}-H_{k+1}^{i} h^{i}\left(x_{k+1 \mid k}^{i}\right) \\
& =H_{k+1}^{i} \tilde{h}^{i}\left(x_{k+1 \mid k}^{i}\right)+\tilde{H}_{k+1}^{i} h^{i}\left(x_{k+1}\right)+V_{k+1}^{i}
\end{aligned}
$$

Then,

$$
\begin{aligned}
& Q_{k+1}^{e^{i}}=E\left[e_{k+1}^{i}\left(e_{k+1}^{i}\right)^{T}\right] \\
& =H_{k+1}^{i} E\left[\tilde{h}^{i}\left(x_{k+1 k}^{i}\right)\left(\tilde{h}^{i}\left(x_{k+1 k}^{i}\right)\right)^{T} \mid Y_{k}^{i}\right]\left(H_{k+1}^{i}\right)^{T} \\
& +\Omega_{k+1}^{\tilde{H}^{i} \tilde{H}^{i}} E\left[h^{i}\left(x_{k+1}\right)\left(h^{i}\left(x_{k+1}\right)\right)^{T} \mid Y_{k}^{i}\right]+R_{k+1}^{V^{i} V^{i}} \\
& =H_{k+1}^{i}\left(\int \tilde{h}^{i}\left(x_{k+1 k}^{i}\right)\left(\tilde{h}^{i}\left(x_{k+1 k}^{i}\right)\right)^{T} N_{1}^{k+1} d x_{k+1}\right)\left(H_{k+1}^{i}\right)^{T} \\
& +\Omega_{k+1}^{\tilde{H}^{i} \tilde{H}^{i}}\left(\int h^{i}\left(x_{k+1}\right)\left(h^{i}\left(x_{k+1}\right)\right)^{T} N_{1}^{k+1} d x_{k+1}\right)+R_{k+1}^{V^{i} V^{i}}
\end{aligned}
$$

The innovation covariance is calculated as follows:

$Q_{k+1}^{e^{i} e^{j}}=E\left[e_{k+1}^{i}\left(e_{k+1}^{j}\right)^{T}\right]$

$=H_{k+1}^{i} E\left[\tilde{h}^{i}\left(x_{k+1 \mid k}^{i}\right)\left(\tilde{h}^{j}\left(x_{k+1 k}^{j}\right)\right)^{T} \mid Y_{k}^{i}, Y_{k}^{j}\right]\left(H_{k+1}^{j}\right)^{T}$

$+\Omega_{k+1}^{\tilde{H}^{i} \tilde{H}^{j}} E\left[h^{i}\left(x_{k+1}\right)\left(h^{j}\left(x_{k+1}\right)\right)^{T} \mid Y_{k}^{i}, Y_{k}^{j}\right]+R_{k+1}^{V^{i} V^{j}}$

$=H_{k+1}^{i}\left(\int \tilde{h}^{i}\left(x_{k+1 \mid k}^{i}\right)\left(\tilde{h}^{j}\left(x_{k+1 \mid k}^{j}\right)\right)^{T} N_{2}^{k+1} d\left[\begin{array}{c}x_{k+1} \\ x_{k+1}\end{array}\right]\right)\left(H_{k+1}^{j}\right)^{T}$

$+\Omega_{k+1}^{\tilde{H}^{i} \tilde{H}^{j}}\left(\int h^{i}\left(x_{k+1}\right)\left(h^{j}\left(x_{k+1}\right)\right)^{T} N_{2}^{k+1} d\left[\begin{array}{c}x_{k+1} \\ x_{k+1}\end{array}\right]\right)+R_{k+1}^{V^{i} V^{j}}$

Lemma 3: For systems (5) and (6), the local filter is designed as follows:

$$
\begin{gathered}
x_{k+1 \mid k+1}^{i}=x_{k+1 \mid k}^{i}+K_{k+1 \mid k}^{x^{i} y^{i}} e_{k+1}^{i} \\
K_{k+1 \mid k}^{x^{i} y^{i}}=P_{k+1 \mid k}^{x^{i} y^{i}}\left(Q_{k+1}^{e^{i}}\right)^{-1}
\end{gathered}
$$

where,

$$
\begin{aligned}
& P_{k+1 \mid k}^{x^{i} y^{i}}=E\left[\left(x_{k+1}-x_{k+1 \mid k}^{i}\right)\left(y_{k+1}^{i}-y_{k+1 \mid k}^{i}\right)^{T} \mid Y_{k}^{i}\right] \\
& =E\left[\left(x_{k+1}-x_{k+1 \mid k}^{i}\right)\left(\begin{array}{l}
H_{k+1}^{i} \tilde{h}^{i}\left(x_{k+1 \mid k}^{i}\right) \\
+\tilde{H}_{k+1}^{i} h^{i}\left(x_{k+1}\right)+V_{k+1}^{i}
\end{array}\right)^{T} \mid Y_{k}^{i}\right] \\
& =E\left[\tilde{x}_{k+1 \mid k}^{i}\left(\tilde{h}^{i}\left(x_{k+1 \mid k}^{i}\right)\right)^{T} \mid Y_{k}^{i}\right]\left(H_{k+1}^{i}\right)^{T} \\
& =\left(\int \tilde{x}_{k+1 \mid k}^{i}\left(\tilde{h}^{i}\left(x_{k+1 \mid k}^{i}\right)\right)^{T} N_{1}^{k+1} d x_{k+1}\right)\left(H_{k+1}^{i}\right)^{T}
\end{aligned}
$$

The covariance is calculated as

$$
\begin{aligned}
P_{k+1 \mid k+1}^{x^{i} x^{i}} & =E\left[\begin{array}{l}
\left(x_{k+1}-x_{k+1 \mid k}^{i}-K_{k+1 \mid k}^{x^{i} y^{i}} e_{k+1}^{i}\right) \\
\left(x_{k+1}-x_{k+1 \mid k}^{i}-K_{k+1 k}^{x^{i} y^{i}} e_{k+1}^{i}\right)^{T} \mid Y_{k}^{i}
\end{array}\right] \\
= & P_{k+1 \mid k}^{x^{i^{i} x^{i}}}-P_{k+1 \mid k}^{x^{i} e^{i}}\left(K_{k+1 k}^{x^{i} y^{i}}\right)^{T}-K_{k+1 \mid k}^{x^{i} y^{i}}\left(P_{k+1 \mid k}^{x^{i} e^{i}}\right)^{T} \\
& +K_{k+1 \mid k}^{x^{i} y^{i}} Q_{k+1}^{i}\left(K_{k+1 \mid k}^{x^{i} y^{i}}\right)^{T}
\end{aligned}
$$

Considering $K_{k+1 \mid k}^{x^{i} y^{i}}=P_{k+1 \mid k}^{x^{i} y^{i}}\left(Q_{k+1}^{e^{i}}\right)^{-1}$, one can obtain

$$
P_{k+1 \mid k}^{x^{i} y^{i}}=K_{k+1 \mid k}^{x^{i} y^{i}} Q_{k+1}^{e^{i}}
$$

Take the above formula into the covariance matrix to get:

$$
\begin{aligned}
& \left.P_{k+1 \mid k+1}^{x^{i} x^{i}}=E\left[\begin{array}{l}
\left(x_{k+1}-x_{k+1 \mid k}^{i}-K_{k+1 k}^{x^{i} y^{i}} e_{k+1}^{i}\right. \\
\left(x_{k+1}-x_{k+1 \mid k}^{i}-K_{k+1 \mid k}^{x^{i} y^{i}} e_{k+1}^{i}\right.
\end{array}\right)^{T} \mid Y_{k}^{i}\right] \\
& =P_{k+1 \mid k}^{x^{i} x^{i}}-K_{k+1 \mid k}^{x^{i} y^{i}} Q_{k+1}^{e^{i}}\left(K_{k+1 \mid k}^{x^{i} y^{i}}\right)^{T}
\end{aligned}
$$

The cross-covariance matrix is calculated as follows 


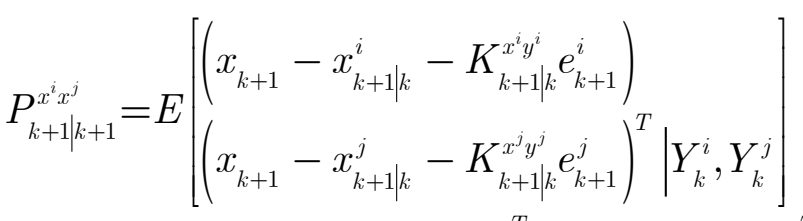

$$
\begin{aligned}
& =P_{k+1 \mid k}^{x^{i} x^{j}}-P_{k+1 \mid k}^{x^{i} y^{j}}\left(K_{k+1 \mid k}^{x^{j} y^{j}}\right)^{T}-K_{k+1 \mid k}^{x^{i} y^{i}}\left(P_{k+1 \mid k}^{x^{j} y^{i}}\right)^{T} \\
& +K_{k+1 \mid k}^{x^{i} y^{i}} Q_{k+1}^{e^{i} e^{j}}\left(K_{k+1 \mid k}^{x^{j} y^{j}}\right)^{T}
\end{aligned}
$$

where,

$$
\begin{aligned}
& P_{k+1 \mid k}^{x^{i} y^{j}}=E\left[\left(x_{k+1}-x_{k+1 \mid k}^{i}\right)\left(y_{k+1}^{j}-y_{k+1 \mid k}^{j}\right)^{T} \mid Y_{k}^{i}, Y_{k}^{j}\right] \\
& =E\left[\left(x_{k+1}-x_{k+1 \mid k}^{i}\right)\left(\begin{array}{l}
\left.H_{k+1}^{j} \tilde{h}^{j}\left(x_{k+1 \mid k}\right)+\tilde{H}\right)^{T} h_{k+1}^{j}\left(x_{k+1}\right)+V_{k+1}^{j} \\
h^{T}
\end{array}\right]\right. \\
& =E\left[\tilde{x}_{k+1 \mid k}^{i}\left(\tilde{h}^{j}\left(x_{k+1 \mid k}\right)\right)^{T}\right]\left(H_{k+1}^{j}\right)^{T} \\
& =\int \tilde{x}_{k+1 \mid k}^{i}\left(\tilde{h}^{j}\left(x_{k+1 \mid k}\right)\right)^{T} N_{2}^{k+1} d\left[\begin{array}{l}
x_{k+1} \\
x_{k+1}
\end{array}\right]\left(H_{k+1}^{j}\right)^{T}
\end{aligned}
$$

$P_{k+1 \mid k}^{x^{j} y^{i}}$ can be obtained similar to the solving process of $P_{k+1 \mid k}^{x^{i} y^{j}}$

Theorem 2: For systems (5) and (6), the corrected state is calculated as follows:

$$
\begin{aligned}
x_{k+1 \mid k+1}^{f} & =x_{k+1 \mid k}^{f}+L_{k+1}\left(\hat{X}_{k+1 \mid k+1}-e x_{k+1 \mid k}^{f}\right) \\
& =\left(I_{n}-L_{k+1} e\right) x_{k+1 \mid k}^{f}+L_{k+1} \hat{X}_{k+1 \mid k+1} \\
P_{k+1 \mid k+1}^{f} & =E\left[\tilde{x}_{k+1 \mid k+1}^{f}\left(\tilde{x}_{k+1 \mid k+1}^{f}\right)^{T} \mid Y_{k+1}\right] \\
= & E\left[\left(\left(I_{n}-L_{k+1} e\right) \tilde{x}_{k+1 \mid k}^{f}+L_{k+1} \tilde{X}_{k+1 \mid k+1}\right)(\bullet)^{T}\right] \\
= & \left(I_{n}-L_{k+1} e\right) P_{k+1 \mid k}^{f}\left(I_{n}-L_{k+1} e\right)^{T} \\
+ & \left(I_{n}-L_{k+1} e\right) P_{k+1 \mid k, k+1}^{x^{f} X} L_{k+1}^{T}(\bullet)^{T} \\
+ & L_{k+1} \Xi_{k+1 \mid k+1} L_{k+1}^{T}
\end{aligned}
$$

$$
\begin{aligned}
& L_{k+1}=\left(P_{k+1 \mid k}^{f} e^{T}-P_{k+1 \mid k, k+1}^{x^{f} X}\right) \\
& \left(e P_{k+1 \mid k}^{f} e^{T}-e P_{k+1 \mid k, k+1}^{x^{f} X}-\left(P_{k+1 k, k+1}^{x^{f} X}\right)^{T} e^{T}+\Xi_{k+1 \mid k+1}\right)^{-1}
\end{aligned}
$$

The proof process of the above three formulas is shown in Appendix 2.

\section{THE NUMERICAL REALIZATION}

In order to facilitate computer simulation, according to the design process of predictors and filters in Appendix 1 and Appendix 2, based on the third-order spherical-radial rule, the numerical implementation format is given as follows.

\section{Design of local predictor:}

Assuming the information at $k-1$ epoch is known, let

$$
\eta_{1}=\left[\begin{array}{c}
x_{k \mid k-1}^{i} \\
x_{k \mid k-1}^{j}
\end{array}\right], \Gamma_{1}=\left[\begin{array}{cc}
P_{k \mid k-1}^{x^{i} x^{i}} & P_{k \mid k-1}^{x^{i} x^{j}} \\
\left(P_{k \mid k-1}^{x^{i} x^{j}}\right) & P_{k \mid k-1}^{x^{j} x^{j}}
\end{array}\right]
$$

1) Factorize

$$
\begin{gathered}
\boldsymbol{P}_{k \mid k-1}^{i}=\boldsymbol{S}_{k \mid k-1}^{i}\left(\boldsymbol{S}_{k \mid k-1}^{i}\right)^{T} \\
\Gamma_{1}=M_{1} M_{1}^{T}
\end{gathered}
$$

2) Evaluate the cubature points

$$
\begin{aligned}
& \chi_{k \mid k-1}^{i, l}=S_{k \mid k-1}^{i} \xi_{l}+x_{k \mid k-1}^{i}, l=1,2, \cdots, 2(n) \\
& {\left[\begin{array}{c}
\breve{\chi}_{k \mid k-1}^{i, l} \\
\breve{\chi}_{k \mid k-1}^{j, l}
\end{array}\right]=M_{1} \varsigma_{l}+\eta_{1}, l=1,2, \cdots, 2(n+n)}
\end{aligned}
$$

3) Propagate the cubature points

$$
\begin{gathered}
\boldsymbol{\mu}_{k \mid k-1}^{i, l}=\boldsymbol{f}\left(\chi_{k \mid k-1}^{i, l}\right), l=1,2, \cdots, 2 n \\
\breve{\boldsymbol{\mu}}_{k \mid k-1}^{i, l}=\boldsymbol{f}\left(\breve{\chi}_{k \mid k-1}^{i, l}\right), l=1,2, \cdots, 2(n+n) \\
\breve{\boldsymbol{\mu}}_{k \mid k-1}^{j, l}=\boldsymbol{f}\left(\breve{\chi}_{k \mid k-1}^{j, l}\right), l=1,2, \cdots, 2(n+n) \\
\breve{\sigma}_{k \mid k-1}^{i, l}=\boldsymbol{h}^{i}\left(\breve{\chi}_{k \mid k-1}^{i, l}\right), l=1,2, \cdots, 2(n+n) \\
\breve{\sigma}_{k \mid k-1}^{j, l}=\boldsymbol{h}^{j}\left(\breve{\chi}_{k \mid k-1}^{j, l}\right), l=1,2, \cdots, 2(n+n)
\end{gathered}
$$


4) Calculate the local prediction mean and covariance

$$
\boldsymbol{x}_{k+1 \mid k-1}^{i}=\Phi_{k}\left(\frac{1}{2 n} \sum_{l=1}^{2 n} \mu_{k \mid k-1}^{i, l}\right)
$$

Let

$$
\begin{aligned}
& \alpha=\frac{1}{2(n+n)} \sum_{l=1}^{2(n+n)} \breve{\boldsymbol{\mu}}_{k \mid k-1}^{i, l}\left(\breve{\sigma}_{k \mid k-1}^{i, l}\right)^{T} \\
& \beta=\frac{1}{2(n+n)} \sum_{l=1}^{2(n+n)} \breve{\mu}_{k \mid k-1}^{i, l} \\
& \chi=\frac{1}{2(n+n)} \sum_{l=1}^{2(n+n)} \sigma_{k \mid k-1}^{i, l} \\
& P_{k+1, k \mid k-1}^{x^{i} y^{i}}=\Phi_{k}\left(\alpha-\beta \chi^{T}\right)\left(H_{k}^{i}\right)^{T} \\
& +\Omega_{k}^{\tilde{\Phi} \tilde{H}^{i}} \alpha+S_{k}^{W V^{i}} \\
& \varepsilon=\frac{1}{2(n+n)} \sum_{l=1}^{2(n+n)} \breve{\boldsymbol{\mu}}_{k \mid k-1}^{i, l}\left(\breve{\boldsymbol{\mu}}_{k \mid k-1}^{j, l}\right)^{T} \\
& \kappa=\frac{1}{2(n+n)} \sum_{i=1}^{2(n+n)} \breve{\boldsymbol{\mu}}_{k \mid k-1}^{j, l} \\
& \lambda=\frac{1}{2(n+n)} \sum_{l=1}^{2(n+n)} \bar{\sigma}_{k \mid k-1}^{i, l}\left(\bar{\sigma}_{k \mid k-1}^{j, l}\right)^{T} \\
& \widehat{\chi}=\frac{1}{2(n+n)} \sum_{l=1}^{2(n+n)} \breve{\sigma}_{k \mid k-1}^{j, l} \\
& \nu=\frac{1}{2(n+n)} \sum_{l=1}^{2(n+n)} \widetilde{\sigma}_{k \mid k-1}^{i, l}\left(\breve{\boldsymbol{\mu}}_{k \mid k-1}^{j, l}\right)^{T} \\
& o=\frac{1}{2(n+n)} \sum_{l=1}^{2(n+n)} \breve{\mu}_{k \mid k-1}^{i, l}\left(\breve{\sigma}_{k \mid k-1}^{j, l}\right)^{T} \\
& \Psi_{1}=\Phi_{k}\left(\varepsilon-\beta \kappa^{T}\right) \Phi_{k}^{T} \\
& +K_{k+1, k \mid k-1}^{x^{i} y^{i}} H_{k}^{i}\left(\lambda-\chi \widehat{\chi}^{T}\right)\left(K_{k+1, k \mid k-1}^{x^{j} y^{j}} H_{k}^{j}\right)^{T} \\
& -K_{k+1, k \mid k-1}^{x^{i} y^{i}} H_{k}^{i}\left(\nu-\chi \kappa^{T}\right) \Phi_{k}^{T} \\
& -\Phi_{k}\left(o-\beta \chi^{T}\right)\left(K_{k+1, k \mid k-1}^{x^{j} y^{j}} H_{k}^{j}\right)^{T}
\end{aligned}
$$

$$
\begin{aligned}
l_{2}= & \Omega_{k}^{\tilde{\Phi} \tilde{\Phi}} \varepsilon-K_{k+1, k \mid k-1}^{x^{i} y^{i}} \Omega_{k}^{\tilde{H}^{i} \tilde{\Phi}} \nu \\
& -\Omega_{k}^{\tilde{\Phi} \tilde{H}^{j}} o\left(K_{k+1, k \mid k-1}^{x^{j} y^{j}}\right)^{T} \\
& +K_{k+1, k \mid k-1}^{x^{i} y^{i}} \Omega_{k}^{\tilde{H}^{i} \tilde{H}^{j}} \lambda\left(K_{k+1, k \mid k-1}^{x^{j} y^{j}}\right)^{T}
\end{aligned}
$$

$$
\begin{aligned}
& l_{3}=Q_{k}^{W}+K_{k+1, k \mid k-1}^{x^{i} y^{i}} R_{k}^{V^{i} V^{j}}\left(K_{k+1, k \mid k-1}^{x^{j} y^{j}}\right)^{T} \\
& -S_{k}^{W V^{j}}\left(K_{k+1, k \mid k-1}^{x^{j} y^{j}}\right)^{T}-K_{k+1, k \mid k-1}^{x^{i} y^{i}}\left(S_{k}^{W V^{i}}\right)^{T}
\end{aligned}
$$

Taking (47) and (51) into (8) and (9), one can get the onestep prediction mean and gain matrix of local predictor. Taking (58)-(60) into (13) yields the one-step prediction covariance matrix.

Design of distributed fusion predictor:

Assuming that the information at $k-1$ epoch is known, let

$$
\eta_{2}=\left[\begin{array}{c}
x_{k \mid k-1}^{f} \\
X_{k \mid k-1}
\end{array}\right], \Gamma_{2}=\left[\begin{array}{cc}
P_{k \mid k-1}^{x^{f} x^{f}} & P_{k \mid k-1}^{x^{f} X} \\
\left(P_{k \mid k-1}^{x^{f} X}\right)^{T} & \Xi_{k \mid k-1}
\end{array}\right]
$$

1) Factorize

$$
\begin{gathered}
\boldsymbol{P}_{k \mid k-1}^{f}=\boldsymbol{S}_{k \mid k-1}^{f}\left(\boldsymbol{S}_{k \mid k-1}^{f}\right)^{T} \\
\Gamma_{2}=M_{2} M_{2}^{T}
\end{gathered}
$$

2) Evaluate the cubature points

$$
\begin{gathered}
\chi_{k \mid k-1}^{f, l}=S_{k \mid k-1}^{f} \xi_{l}+x_{k \mid k-1}^{f}, l=1,2, \cdots, 2(n) \\
{\left[\begin{array}{c}
\breve{\chi}_{k \mid k-1}^{f, l} \\
\check{\chi}_{k \mid k-1}^{X, l}
\end{array}\right]=M_{2} \zeta_{l}+\eta_{2}, l=1,2, \cdots, 2\left(n+n^{*} N\right)}
\end{gathered}
$$

3) Propagate the cubature points

$$
\begin{gathered}
\boldsymbol{\mu}_{k \mid k-1}^{f, l}=\boldsymbol{f}\left(\chi_{k \mid k-1}^{f, l}\right), l=1,2, \cdots, 2 n \\
\sigma_{k \mid k-1}^{f, l}=\boldsymbol{h}\left(\chi_{k \mid k-1}^{f, l}\right), l=1,2, \cdots, 2 n \\
\breve{\boldsymbol{\mu}}_{k \mid k-1}^{f, l}=\boldsymbol{f}\left(\breve{\chi}_{k \mid k-1}^{f, l}\right), l=1,2, \cdots, 2\left(n+n^{*} N\right) \\
\breve{\boldsymbol{\mu}}_{k \mid k-1}^{X, l}=\boldsymbol{f}\left(\breve{\chi}_{k \mid k-1}^{X, l}\right), l=1,2, \cdots, 2\left(n+n^{*} N\right)
\end{gathered}
$$




$$
\breve{\sigma}_{k \mid k-1}^{X, l}=\boldsymbol{h}\left(\breve{\chi}_{k \mid k-1}^{X, l}\right), l=1,2, \cdots, 2(n+n * N)
$$

4) Calculate the mean and covariance after fusion

$$
\begin{gathered}
x_{k+1 \mid k}^{f}=\left(I_{n}-L_{k+1} e\right) \Phi_{k}\left(\frac{1}{2 n} \sum_{l=1}^{2 n} \boldsymbol{\mu}_{k \mid k-1}^{f, l}\right)+L_{k+1} \hat{X}_{k+1 \mid k} \\
\bar{\alpha}=\frac{1}{2 n} \sum_{l=1}^{2 n} \boldsymbol{\mu}_{k \mid k-1}^{f, l}\left(\boldsymbol{\mu}_{k \mid k-1}^{f, l}\right)^{T} \\
\bar{\beta}=\frac{1}{2 n} \sum_{l=1}^{2 n} \boldsymbol{\mu}_{k \mid k-1}^{f, l} \\
P_{k+1 \mid k}^{f}=\left(\begin{array}{c}
\left.I_{n}-L_{k+1} e\right) \times \\
\Phi_{k}\left(\begin{array}{l}
\bar{\alpha} \\
-\overline{\beta \beta}
\end{array} \bar{\beta}^{T} \Phi_{k}^{T}\right. \\
+\Omega_{k}^{\tilde{\Phi} \tilde{\Phi}} \bar{\alpha}+Q_{k}^{W W}
\end{array}\right\}\left(I_{n}-L_{k+1} e\right)^{T} \\
+L_{k+1} \Xi_{k+1 \mid k} L_{k+1}^{T}+\Lambda+\Lambda^{T}
\end{gathered}
$$$$
\bar{\chi}=\frac{1}{2\left(n+n^{*} N\right)} \sum_{l=1}^{2\left(n+n^{*} N\right)} \breve{\boldsymbol{\mu}}_{k \mid k-1}^{f, l}\left(\breve{\boldsymbol{\mu}}_{k \mid k-1}^{X, l}\right)^{T}
$$$$
\bar{\delta}=\frac{1}{2\left(n+n^{*} N\right)} \sum_{l=1}^{2\left(n+n^{*} N\right)} \breve{\boldsymbol{\mu}}_{k \mid k-1}^{f, l}
$$$$
\bar{\varepsilon}=\frac{1}{2\left(n+n^{*} N\right)} \sum_{l=1}^{2\left(n+n^{*} N\right)} \breve{\boldsymbol{\mu}}_{k \mid k-1}^{X, l}
$$$$
\bar{\phi}=\frac{1}{2\left(n+n^{*} N\right)} \sum_{l=1}^{2\left(n+n^{*} N\right)} \breve{\sigma}_{k \mid k-1}^{X, l}
$$$$
\bar{\varphi}=\frac{1}{2\left(n+n^{*} N\right)} \sum_{l=1}^{2\left(n+n^{*} N\right)} \breve{\boldsymbol{\mu}}_{k \mid k-1}^{f, l}\left(\sigma_{k \mid k-1}^{X, l}\right)^{T}
$$$$
\mathrm{A}=\Phi_{k}\left(\bar{\chi}-\bar{\delta} \bar{\varepsilon}^{T}\right) \Psi_{k}^{T}
$$$$
-\Phi_{k}\left(\bar{\chi}-\overline{\delta \phi}^{T}\right)\left(K_{k+1, k \mid k-1}^{x y} H_{k}\right)^{T}
$$$$
\mathrm{B}=\Omega_{k}^{\tilde{\Phi} \tilde{\Psi}} \bar{\chi}-\Omega_{k}^{\tilde{\Phi} \tilde{H}} \bar{\varphi}\left(K_{k+1, k \mid k-1}^{x y}\right)^{T}
$$

$$
C=\Phi_{k}\left(\bar{\alpha}-\bar{\beta} \bar{\beta}^{T}\right) \Phi_{k}^{T}+\Omega_{k}^{\tilde{\Phi} \tilde{\Phi}} \bar{\alpha}
$$

Taking the values of $A, B, C$ into $\Lambda$ and $L_{k+1}$ yields the prediction mean and covariance.

\section{Calculation of innovation:}

Assuming the information at $k$ epoch is known, let

$$
\eta_{3}=\left[\begin{array}{c}
x_{k \mid k-1}^{i} \\
x_{k \mid k-1}^{j}
\end{array}\right], \Gamma_{3}=\left[\begin{array}{cc}
P_{k \mid k-1}^{x^{i} x^{i}} & P_{k \mid k-1}^{x^{i} x^{j}} \\
\left(P_{k \mid k-1}^{x^{i} x^{j}}\right)^{T} & P_{k \mid k-1}^{x^{j} x^{j}}
\end{array}\right]
$$

1) Factorize

$$
\begin{gathered}
\boldsymbol{P}_{k+1 \mid k}^{i}=\boldsymbol{S}_{k+1 \mid k}^{i}\left(\boldsymbol{S}_{k+1 \mid k}^{i}\right)^{T} \\
\Gamma_{3}=M_{3} M_{3}^{T}
\end{gathered}
$$

2) Evaluate the cubature points

$$
\begin{gathered}
\chi_{k+1 \mid k}^{i, l}=S_{k+1 \mid k}^{i, l} \xi_{l}+x_{k+1 \mid k}^{i}, l=1,2, \cdots, 2(n) \\
{\left[\begin{array}{c}
\breve{\chi}_{k+1 \mid k}^{i, l} \\
\bar{\chi}_{k+1 \mid k}^{j, l}
\end{array}\right]=M_{3} \zeta_{l}+\eta_{3}, l=1,2, \cdots, 2(n+n)}
\end{gathered}
$$

3) Propagate the cubature points

$$
\begin{gathered}
\sigma_{k+1 \mid k}^{i, l}=\boldsymbol{h}^{i}\left(\chi_{k+1 \mid k}^{i, l}\right), i=1,2, \cdots, 2 n \\
\breve{\sigma}_{k+1 \mid k}^{i, l}=\boldsymbol{h}^{i}\left(\breve{\chi}_{k+1 \mid k}^{i, l}\right), l=1,2, \cdots, 2(n+n) \\
\breve{\sigma}_{k+1 \mid k}^{j, l}=\boldsymbol{h}^{j}\left(\breve{\chi}_{k+1 \mid k}^{j, l}\right), l=1,2, \cdots, 2(n+n)
\end{gathered}
$$

4) Calculate the innovation and covariance

$$
\begin{gathered}
y_{k+1 \mid k}^{i}=H_{k+1}^{i}\left(\frac{1}{2 n} \sum_{l=1}^{2 n} \sigma_{k+1 \mid k}^{i, l}\right) \\
Q_{k+1}^{e^{i}}=H_{k+1}^{i}\left(\begin{array}{l}
\frac{1}{2 n} \sum_{l=1}^{2 n} \sigma_{k+1 \mid k}^{i, l}\left(\sigma_{k+1 \mid k}^{i, l}\right)^{T} \\
\left.-\left(\frac{1}{2 n} \sum_{l=1}^{2 n} \sigma_{k+1 \mid k}^{i, l}\right)\left(\frac{1}{2 n} \sum_{l=1}^{2 n} \sigma_{k+1 \mid k}^{i, l}\right)^{T}\right) \times \\
\left(H_{k+1}^{i}\right)^{T}+\Omega_{k+1}^{\tilde{H}^{i} \tilde{H}^{i}}\left(\frac{1}{2 n} \sum_{l=1}^{2 n} \sigma_{k+1 \mid k}^{i, l}\left(\sigma_{k+1 \mid k}^{i, l}\right)^{T}\right)+R_{k+1}^{V^{i} V^{i}}
\end{array}\right.
\end{gathered}
$$


The innovation cross-covariance is

$$
\begin{aligned}
& Q_{k+1}^{e^{i} e^{j}}=H_{k+1}^{i}\left(\begin{array}{l}
\frac{1}{2(n+n)} \sum_{l=1}^{2(n+n)} \breve{\sigma}_{k+1 \mid k}^{i, l}\left(\breve{\sigma}_{k+1 \mid k}^{j, l}\right)^{T} \\
-\left(\frac{1}{2 n} \sum_{l=1}^{2(n+n)} \breve{\sigma}_{k+1 \mid k}^{i, l}\right)\left(\frac{1}{2 n} \sum_{l=1}^{2(n+n)} \breve{\sigma}_{k+1 \mid k}^{j, l}\right)^{T}
\end{array}\right) \times \\
& \left(H_{k+1}^{j}\right)^{T}+\Omega_{k+1}^{\tilde{H}^{i} \tilde{H}^{j}}\left(\frac{1}{2 n} \sum_{l=1}^{2(n+n)} \breve{\sigma}_{k+1 \mid k}^{i, l}\left(\breve{\sigma}_{k+1 \mid k}^{j, l}\right)^{T}\right)+R_{k+1}^{V^{i} V^{j}}
\end{aligned}
$$

\section{Design of local filter:}

$$
\eta_{4}=\left[\begin{array}{c}
x_{k+1 \mid k}^{i} \\
x_{k+1 \mid k}^{j}
\end{array}\right], \Gamma_{4}=\left[\begin{array}{cc}
P_{k+1 \mid k}^{x^{i} x^{i}} & P_{k+1 \mid k}^{x^{i} x^{j}} \\
\left(P_{k+1 \mid k}^{x^{i} x^{j}}\right)^{T} & P_{k+1 \mid k}^{x^{j} x^{j}}
\end{array}\right]
$$

1) Factorize

$$
\begin{gathered}
\boldsymbol{P}_{k+1 \mid k}^{i}=\boldsymbol{S}_{k+1 \mid k}^{i}\left(\boldsymbol{S}_{k+1 \mid k}^{i}\right)^{T} \\
\Gamma_{4}=M_{4} M_{4}^{T}
\end{gathered}
$$

2) Evaluate the cubature points

$$
\begin{gathered}
\chi_{k+1 \mid k}^{i, l}=S_{k+1 \mid k}^{i} \xi_{l}+x_{k+1 \mid k}^{i}, l=1,2, \cdots, 2 n \\
{\left[\begin{array}{c}
\breve{\chi}_{k+1 \mid k}^{i, l} \\
\bar{\chi}_{k+1 \mid k}^{j, l}
\end{array}\right]=M_{3} \varsigma_{l}+\eta_{3}, l=1,2, \cdots, 2(n+n)}
\end{gathered}
$$

3) Propagate the cubature points

$$
\begin{gathered}
\sigma_{k+1 \mid k}^{i, l}=h\left(\chi_{k+1 \mid k}^{i, l}\right), l=1,2, \cdots, 2 n \\
\breve{\sigma}_{k+1 \mid k}^{j, l}=\boldsymbol{h}\left(\breve{\chi}_{k+1 \mid k}^{j, l}\right), l=1,2, \cdots, 2(n+n)
\end{gathered}
$$

4) Calculate the covariance

$$
\begin{aligned}
P_{k+1 \mid k}^{x^{i} y^{i}} & \left(\begin{array}{l}
\frac{1}{2 n} \sum_{l=1}^{2 n} \chi_{k+1 \mid k}^{i, l}\left(\sigma_{k+1 \mid k}^{i, l}\right)^{T} \\
-\left(\frac{1}{2 n} \sum_{l=1}^{2 n} \chi_{k+1 \mid k}^{i, l}\right)\left(\frac{1}{2 n} \sum_{l=1}^{2 n} \sigma_{k+1 \mid k}^{i, l}\right)^{T}
\end{array}\right)\left(H_{k+1}^{i}\right)^{T}
\end{aligned}
$$

$$
P_{k+1 \mid k}^{x^{i} y^{j}}=\left(\begin{array}{l}
\frac{1}{2(2 n)} \sum_{l=1}^{2(2 n)} \widetilde{\chi}_{k+1 \mid k}^{i, l}\left(\breve{\sigma}_{k+1 \mid k}^{j, l}\right)^{T} \\
-\left(\frac{1}{2(2 n)} \sum_{l=1}^{2(2 n)} \widetilde{\chi}_{k+1 \mid k}^{i, l}\right)\left(\frac{1}{2(2 n)} \sum_{l=1}^{2(2 n)} \breve{\sigma}_{k+1 \mid k}^{j, l}\right)^{T}
\end{array}\right)\left(H_{k+1}^{j}\right)^{T}
$$

Fusion filter design:

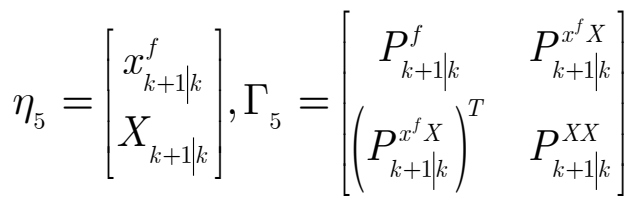

$$
\eta_{6}=\left[\begin{array}{c}
x_{k \mid k-1}^{f} \\
x_{k \mid k-1}^{i}
\end{array}\right], \Gamma_{6}=\left[\begin{array}{cc}
P_{k \mid k-1}^{f} & P_{k \mid k-1}^{x^{f} x^{i}} \\
\left(P_{k \mid k-1}^{x^{f} x^{i}}\right)^{T} & P_{k \mid k-1}^{x^{i} x^{i}}
\end{array}\right]
$$

1) Factorize

$$
\begin{aligned}
& \Gamma_{5}=M_{5} M_{5}^{T} \\
& \Gamma_{6}=M_{6} M_{6}^{T}
\end{aligned}
$$

2) Evaluate the cubature points

$$
\begin{gathered}
{\left[\begin{array}{c}
\breve{\chi}_{k+1 \mid k}^{f, l} \\
\breve{\chi}_{k+1 \mid k}^{X, l}
\end{array}\right]=M_{5} \zeta_{l}+\eta_{5}, l=1,2, \cdots, 2\left(n+n^{*} N\right)} \\
{\left[\begin{array}{c}
\breve{\chi}_{k+1 \mid k}^{f, l} \\
\bar{\chi}_{k+1 \mid k}^{i, l}
\end{array}\right]=M_{6} \zeta_{l}+\eta_{6}, l=1,2, \cdots, 2(n+n)}
\end{gathered}
$$

3) Propagate the cubature points

$$
\begin{gathered}
\breve{\sigma}_{k+1 \mid k}^{X, l}=h\left(\breve{\chi}_{k+1 \mid k}^{X, l}\right), l=1,2, \cdots, 2\left(n+n^{*} N\right) \\
\breve{\boldsymbol{\mu}}_{k+1 \mid k}^{f, l}=\boldsymbol{f}\left(\breve{\chi}_{k+1 \mid k}^{f, l}\right), l=1,2, \cdots, 2(n+n)
\end{gathered}
$$

$$
\begin{aligned}
& \breve{\boldsymbol{\mu}}_{k+1 \mid k}^{i, l}=\boldsymbol{f}\left(\breve{\chi}_{k+1 \mid k}^{i, l}\right), l=1,2, \cdots, 2(n+n) \\
& \breve{\sigma}_{k+1 \mid k}^{i, l}=\boldsymbol{h}\left(\breve{\chi}_{k+1 \mid k}^{i, l}\right), l=1,2, \cdots, 2(n+n)
\end{aligned}
$$

4) Calculate the covariance

5) $\operatorname{Let}\left(I_{n}-L_{k+1} e\right)=\overline{\boldsymbol{\psi}}$ 


$$
\begin{aligned}
& \bar{\mu}=\frac{1}{2(n+n N)} \sum_{l=1}^{2(n+n N)} \bar{\chi}_{k+1 \mid k}^{f, l}\left(\breve{\sigma}_{k+1 \mid k}^{X, l}\right)^{T} \\
& \bar{\nu}=\frac{1}{2(n+n N)} \sum_{l=1}^{2(n+n N)} \bar{\chi}_{k+1 \mid k}^{f, l} \\
& \bar{o}=\frac{1}{2(n+n N)} \sum_{l=1}^{2(n+n N)} \breve{\sigma}_{k+1 \mid k}^{X, l} \\
& \bar{\varpi}=\frac{1}{2(n+n)} \sum_{l=1}^{2 n} \breve{\boldsymbol{\mu}}_{k+1 \mid k}^{f, l}\left(\breve{\boldsymbol{\mu}}_{k+1 \mid k}^{i, l}\right)^{T} \\
& \bar{\theta}=\frac{1}{2(n+n)} \sum_{l=1}^{2 n} \breve{\boldsymbol{\mu}}_{k+1 \mid k}^{i, l} \\
& \bar{\vartheta}=\frac{1}{2(n+n)} \sum_{l=1}^{2 n} \breve{\boldsymbol{\mu}}_{k+1 \mid k}^{f, l}\left(\breve{\sigma}_{k+1 \mid k}^{i, l}\right)^{T} \\
& \bar{\rho}=\frac{1}{2(n+n)} \sum_{l=1}^{2 n} \breve{\sigma}_{k+1 \mid k}^{i, l} \\
& \bar{\varpi}=\frac{1}{2(n+n)} \sum_{l=1}^{2 n} \breve{\boldsymbol{\mu}}_{k+1 \mid k}^{f, l}\left(\breve{\boldsymbol{\mu}}_{k+1 \mid k}^{i, l}\right)^{T} \\
& P_{k+1 \mid k, k+1}^{\tilde{x}^{f} \tilde{X}}=P_{k+1 \mid k}^{x^{f} X}-\left(\bar{\mu}-\overline{\nu O}^{T}\right)\left(K_{k+1 \mid k}^{x y} H_{k+1}\right)^{T} \\
& P_{k+1 \mid k}^{x^{f} x^{i}}=\overline{\boldsymbol{\psi}} \Phi_{k}\left(\bar{\varpi}-\bar{\nu} \bar{\theta}^{T}\right) \Phi_{k}^{T} \\
& -\overline{\boldsymbol{\psi}} \Phi_{k}\left(\bar{\vartheta}-\overline{\nu \rho}^{T}\right)\left(K_{k+1, k \mid k-1}^{x^{i} y^{i}} H_{k}^{i}\right)^{T} \\
& +\bar{\psi} \tilde{\Phi}_{k} \bar{\varpi} \tilde{\Phi}_{k}^{T} \\
& -\overline{\boldsymbol{\psi}} \tilde{\Phi}_{k} \bar{\vartheta}\left(K_{k+1, k \mid k-1}^{x^{i} y^{i}} \tilde{H}_{k}^{i}\right)^{T} \\
& +\overline{\boldsymbol{\psi}} Q_{k}^{W}-\overline{\boldsymbol{\psi}} S_{k}^{W V^{i}}\left(K_{k+1, k \mid k-1}^{x^{i} y^{i}}\right)^{T} \\
& +L_{k+1} P_{k+1 \mid k}^{X x^{i}}
\end{aligned}
$$

\section{SimULATION}

In order to verify the effectiveness of the proposed algorithm, a strong nonlinear model is given as follows:

$$
\begin{gathered}
x_{k+1}=\left[\begin{array}{c}
x_{k+1}^{1} \\
x_{k+1}^{2} \\
x_{k+1}^{3}
\end{array}\right]=\Phi_{k}\left[\begin{array}{c}
3 \sin ^{2}\left(5 x_{k}^{2}\right) \\
x_{k}^{1}+e^{-0.05 x_{k}^{3}}+10 \\
0.2 x_{k}^{1}\left(x_{k}^{2}+x_{k}^{3}\right)
\end{array}\right]+\left[\begin{array}{l}
0.1 \\
0.1 \\
0.1
\end{array}\right] \omega_{k} \\
y_{k}^{1}=\Lambda_{k}^{1}\left(\cos \left(x_{k}^{1}\right)+x_{k}^{2} x_{k}^{3}\right)+v_{k}^{1} \\
\Phi_{k}^{2}=\Lambda_{k}^{2}\left(x_{k}^{1}+x_{k}^{2} x_{k}^{3}\right)+v_{k}^{2} \\
I_{3 \times 3}+\xi_{k} d i a g([123) \\
\left.\left.\Lambda_{k}^{1}=1+0.01 \quad 0.01 \quad 0.01\right]\right) \\
\Lambda_{k}^{2}=1+0.3 \zeta_{k} \\
v_{k}^{1}=c_{1} \omega_{k}+\eta_{k} \\
v_{k}^{2}=c_{2} \omega_{k}+\eta_{k} \\
\zeta_{k}=b \xi_{k}+\gamma_{k}
\end{gathered}
$$

where $\omega_{k}, \eta_{k}, \xi_{k}$ and $\gamma_{k}$ are uncorrelated Gaussian white noise, and the covariance is $1,0.5,0.1$ and 0.3 , respectively, and $c_{1}=0.5, c_{2}=0.6, b=1$. The initial value of state is $\bar{x}_{0}=\left[\begin{array}{lll}-0.7 & 1 & 1\end{array}\right]^{T}$, and the initial value of filter is $\bar{x}_{0}=\left[\begin{array}{lll}-0.7 & 1 & 1\end{array}\right]^{T}, \bar{P}_{0}=I_{3 \times 3} .30$ independent Monte Carlo simulations are carried out, and the corresponding simulation results are as follows:
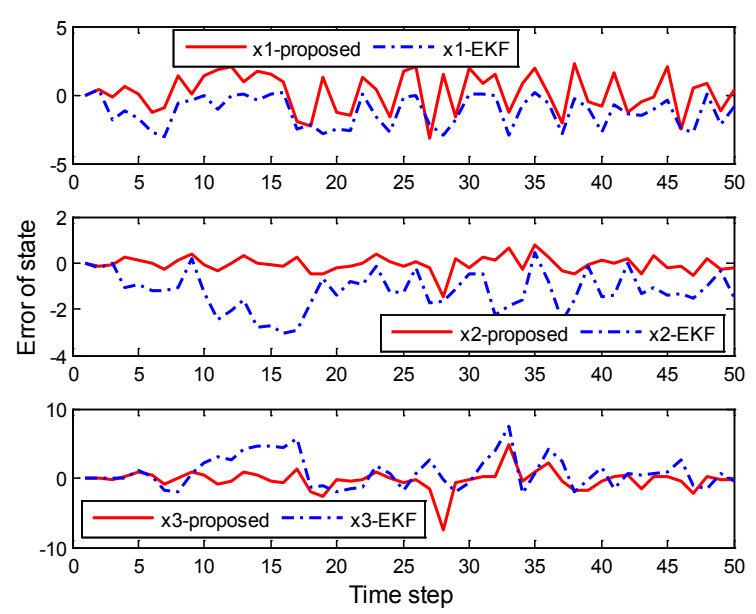

Fig.2. Error of the state. 

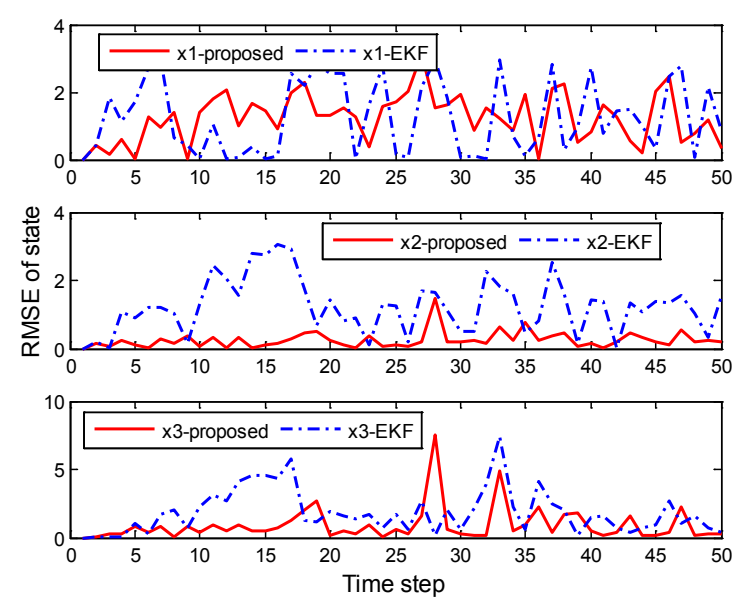

Fig.3. RMSE of the state.

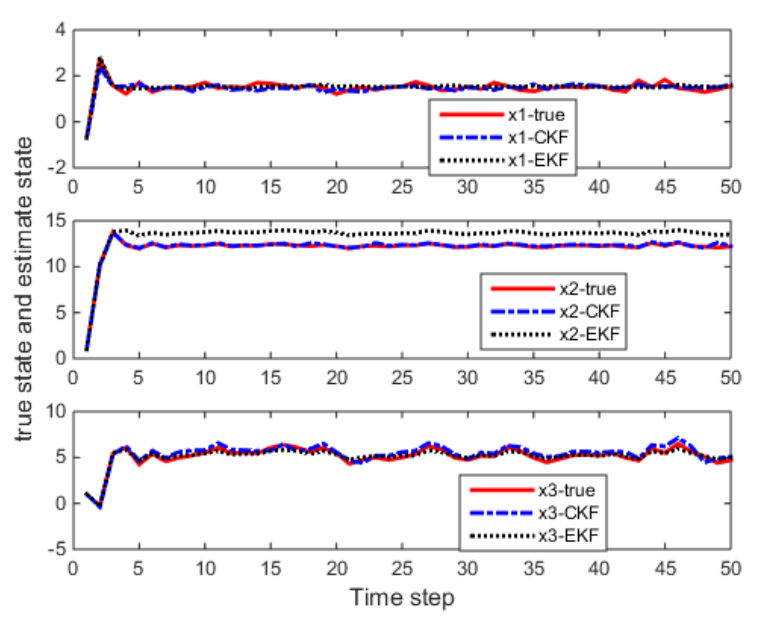

Fig.4. Ture state and estimate state.

It can be seen from Fig.2. and Fig.3. that the proposed algorithm can obtain smaller estimation error and root mean square error when compared with the algorithm in literature to a nonlinear system. It is shown that in a nonlinear system, compared with the conclusion of linear system based on EKF, the nonlinear filter design is directly carried out for the nonlinear system. Higher estimation accuracy can be obtained.

\section{CONCLUSION}

For the nonlinear multi-sensor system with random parameter matrix and correlated noise, the local estimation state is regarded as a measurement and sent to the data processing center to modify the prior information of the data processing center. In this paper, the distributed fusion predictor and filter are derived, and the numerical implementation of the algorithm based on the third-order spherical diameter volume rule is given. The simulation results show that, compared with the EKF algorithm, the estimator designed for nonlinear systems can achieve higher estimation accuracy when dealing with nonlinear problems.

\section{ACKNOWLEDGMENT}

The authors would like to thank the National Natural Science Foundation of China (NSFC), Science Fund for Excellent Young Scholars of Heilongjiang Province and Sichuan Science and Technology Program to sponsor this study. The grant numbers are 6191101340, YQ2020F007, 2021YJ0091, 2021JDRC0092.

\section{REFERENCES}

[1] Scheel, A., Knill, C, Reuter, S., Dietmayer, K. (2016). Multi-sensor multi-object tracking of vehicles using high-resolution radars. In IEEE Intelligent Vehicles Symposium (IV). IEEE, 558-565.

[2] Jing, Z., Pan, H., Li, Y., Dong, P. (2018). Target tracking and multi-sensor fusion with adaptive cubature information filter. In Non-Cooperative Target Tracking, Fusion and Control. Springer, 41-70.

[3] Tao, X., Shi, Z., Li, X. (2018). A new intelliSense strategy based on artificial immune system for multirobot cooperation. Journal of Robotics \& Mechatronics, 30 (1), 128-137.

[4] Zhang, Y.Y., Huang, Y., Liu, Y., Liu, C.X., Liu, P., Zhang, Y. (2020). Human grasp feature learning and object recognition based on multi-sensor information fusion. Robot, 42 (3), 267-277.

[5] Zhang, Q.Y., Zhang, T., Zhao, Z.Y. (2019). Human motion modes recognition based on multi-sensors. Transducer and Microsystem Technologies, 38 (2), 7376.

[6] He, F., Shi, Y.F., Wang, F., Zhao, H.W., Qin, H.B. (2018). Research on GMAW welding process pattern recognition based on multi-sensor and support vector machine. Technology Innovation and Application, 34, 1-7.

[7] Carlson, N.A., Neily, C.M. (1987). Distributed Kalman filter architectures. Final report, AFWAL-TR-87-118 1, Avionics Laboratory, WPAFB, Ohio, US.

[8] Carlson, N.A. (1990). Federated square root filter for decentralized parallel processes. IEEE Transactions on Aerospace and Electronic Systems, 26 (3), 517-525.

[9] Carlson, N.A. (1988). Federated filter for fault-tolerant integrated navigation systems. In IEEE Symposium on Position Location and Navigation (PLANS). IEEE, 110119.

[10] Deng, Z.L. (2012). Information Fusion Estimation Theory and Its Application. Beijing, China: Science Press.

[11] Deng, Z.L., Gao, Y., Mao, L., Li, Y., Hao, G. (2005). New approach to information fusion steady-state Kalman filtering. Automatica, 41 (10), 1695-1707.

[12] Sun, S.L. (2004). Multi-sensor information fusion white noise filter weighted by scalars based on Kalman predictor. Automatica, 40 (8), 1447-1453.

[13] Julier, S.J., Uhlmann, J.K. (1997). A non-divergent estimation algorithm in the presence of unknown correlations. In American Control Conference (ACC). IEEE, Vol. 4, 2369-2373. 
[14] Deng, Z., Peng, Z., Qi, W., Liu, J., Yuan, G. (2012). Sequential covariance intersection fusion Kalman filter. Information Sciences, 189, 293-309.

[15] Yuan, Y.X., Sun, W. (1997). Optimization Theory and Methods. Beijing, China: Science Press.

[16] Cong, J., Li, Y., Qi, G., Sheng, A. (2016). An order insensitive sequential fast covariance intersection fusion algorithm. In Information Sciences, 367, 28-40.

[17] Lin, H., Sun, S. (2019). Globally optimal sequential and distributed fusion state estimation for multi-sensor systems with cross-correlated noises. Automatica, 101, 128-137.

[18] Sun, S. (2020). Distributed optimal linear fusion predictors and filters for systems with random parameter matrices and correlated noises. IEEE Transactions on Signal Processing, 68, 1064-1074.

\section{Appendix 1}

The proof of Theorem 1:

$$
\begin{aligned}
& \tilde{x}_{k+1 \mid k}^{f}=x_{k+1}-x_{k+1 \mid k}^{f} \\
& =x_{k+1}-\left(I_{n}-L_{k+1} e\right) \Phi_{k} f\left(x_{k \mid k-1}^{f}\right)-L_{k+1} \hat{X}_{k+1 \mid k} \\
& =\left(I_{n}-L_{k+1} e\right) \Phi_{k} \tilde{f}\left(x_{k \mid k-1}^{f}\right)+\left(I_{n}-L_{k+1} e\right) \tilde{\Phi}_{k} f\left(x_{k}\right) \\
& +\left(I_{n}-L_{k+1} e\right) W_{k}+L_{k+1} \tilde{X}_{k+1 \mid k}
\end{aligned}
$$$$
\tilde{x}_{k+1 \mid k}^{f}=x_{k+1}-x_{k+1 k}^{f}
$$$$
=x_{k+1}-\left(I_{n}-L_{k+1} e\right) \Phi_{k} f\left(x_{k \mid k-1}^{f}\right)-L_{k+1} \hat{X}_{k+1 \mid k}
$$$$
=\left(I_{n}-L_{k+1} e\right) \Phi_{k} \tilde{f}\left(x_{k \mid k-1}^{f}\right)+\left(I_{n}-L_{k+1} e\right) \tilde{\Phi}_{k} f\left(x_{k}\right)
$$$$
+\left(I_{n}-L_{k+1} e\right) W_{k}+L_{k+1} \tilde{X}_{k+1 \mid k}
$$

Sorting out local one-step prediction errors yields

$$
\begin{aligned}
& \tilde{X}_{k+1 \mid k}=\left(\Psi_{k} \tilde{f}\left(X_{k \mid k-1}\right)-K_{k+1, k \mid k-1}^{x y} H_{k} \tilde{h}\left(X_{k \mid k-1}\right)\right) \\
& +\left(\tilde{\Psi}_{k} e f\left(x_{k}\right)-K_{k+1, k \mid k-1}^{x y} \tilde{H}_{k} h\left(x_{k}\right)\right)+\left(e W_{k}-K_{k+1, k \mid k-1}^{x y} V_{k}\right)
\end{aligned}
$$

where,

$$
\begin{aligned}
& \tilde{f}\left(X_{k \mid k-1}\right)=\left[\tilde{f}\left(x_{k \mid k-1}^{1}\right), \cdots, \tilde{f}\left(x_{k \mid k-1}^{N}\right)\right]^{T} \\
& \Psi_{k}=\operatorname{diag}\left(\Phi_{k}, \cdots, \Phi_{k}\right) \\
& K_{k+1, k \mid k-1}^{x y}=\operatorname{diag}\left(K_{k+1, k \mid k-1}^{x^{1} y^{1}}, \cdots, K_{k+1, k \mid k-1}^{x^{N} y^{N}}\right) \\
& H_{k}=\operatorname{diag}\left(H_{k}^{1}, \cdots, H_{k}^{N}\right)
\end{aligned}
$$

$$
\begin{gathered}
\tilde{h}\left(X_{k \mid k-1}\right)=\left[\tilde{h}^{1}\left(x_{k \mid k-1}^{1}\right), \cdots, \tilde{h}^{N}\left(x_{k \mid k-1}^{N}\right)\right]^{T} \\
\tilde{\Psi}_{k}=\operatorname{diag}\left(\tilde{\Phi}_{k}, \cdots, \tilde{\Phi}_{k}\right) \\
\tilde{H}_{k}=\operatorname{diag}\left(\tilde{H}_{k}^{1}, \cdots, \tilde{H}_{k}^{N}\right) \\
h\left(x_{k}\right)=\left[h^{1}\left(x_{k}\right), \cdots, h^{N}\left(x_{k}\right)\right]^{T} \\
V_{k}=\left[V_{k}^{1}, \cdots, V_{k}^{N}\right]^{T}
\end{gathered}
$$

Then, the one-step prediction error covariance matrix is

$$
\begin{aligned}
& P_{k+1 \mid k}^{f}=E\left[\tilde{x}_{k+1 \mid k}^{f}\left(\tilde{x}_{k+1 \mid k}^{f}\right)^{T} \mid Y_{k}\right] \\
& =\left(I_{n}-L_{k+1} e\right)\left\{\begin{array}{l}
\Phi_{k} E\left[\tilde{f}\left(x_{k \mid k-1}^{f}\right) \tilde{f}^{T}\left(x_{k \mid k-1}^{f}\right)\right] \Phi_{k}^{T} \\
+\Omega_{k}^{\tilde{\Phi} \tilde{\Phi}} E\left[f\left(x_{k}\right) f^{T}\left(x_{k}\right)\right]+Q_{k}^{W W}
\end{array}\right\} \\
& \times\left(I_{n}-L_{k+1} e\right)^{T}+L_{k+1} \Xi_{k+1 \mid k} L_{k+1}^{T}+\Lambda+\Lambda^{T}
\end{aligned}
$$

where, $\quad \Xi_{k+1 \mid k}=\left(P_{k+1 \mid k}^{x^{i} x^{j}}\right) \quad$ means that all $P_{k+1 \mid k}^{x^{i} x^{j}}, i, j=1, \cdots, N$ are arranged into a square matrix in the order of row $i$ and column $j$.

Let

$$
\begin{gathered}
a a=E\left[\tilde{f}\left(x_{k \mid k-1}^{f}\right) \tilde{f}^{T}\left(X_{k \mid k-1}\right)\right] \\
b b=E\left[\tilde{f}\left(x_{k \mid k-1}^{f}\right) \tilde{h}^{T}\left(X_{k \mid k-1}\right)\right] \\
c c=E\left[f\left(x_{k}\right)\left(e f\left(x_{k}\right)\right)^{T}\right] \\
d d=E\left[f\left(x_{k}\right) h^{T}\left(x_{k}\right)\right]
\end{gathered}
$$

$$
\begin{aligned}
& \Lambda=\left(I_{n}-L_{k+1} e\right)\left\{\begin{array}{l}
\Phi_{k} a a \Psi_{k}^{T}- \\
\left.\Phi_{k} b b\left(K_{k+1, k \mid k-1}^{x y} H_{k}\right)^{T}\right\} L_{k+1}^{T}
\end{array}\right. \\
& +\left(I_{n}-L_{k+1} e\right)\left\{\Omega_{k}^{\tilde{\Phi} \tilde{\Psi}} c c-\Omega_{k}^{\tilde{\Phi} \tilde{H}} d d^{T}\right\} L_{k+1}^{T} \\
& +\left(I_{n}-L_{k+1} e\right)\left\{Q_{k}^{W W} e^{T}-S_{k}^{W V}\left(K_{k+1, k \mid k-1}^{x y}\right)^{T}\right\} L_{k+1}^{T}
\end{aligned}
$$


It is worth mentioning that, according to the definition of matrix $A, B$ later, $\Lambda$ can be rewritten as

$$
\begin{aligned}
\Lambda= & \left(I_{n}-L_{k+1} e\right) A L_{k+1}^{T}+\left(I_{n}-L_{k+1} e\right) B L_{k+1}^{T} \\
& +\left(I_{n}-L_{k+1} e\right)\left\{Q_{k}^{W W} e^{T}-S_{k}^{W V}\left(K_{k+1, k \mid k-1}^{x y}\right)^{T}\right\} L_{k+1}^{T}
\end{aligned}
$$

where,

$$
\begin{gathered}
E\left[\tilde{f}\left(x_{k \mid k-1}^{f}\right) \tilde{f}^{T}\left(x_{k \mid k-1}^{f}\right)\right] \\
=\int \tilde{f}\left(x_{k \mid k-1}^{f}\right) \tilde{f}^{T}\left(x_{k \mid k-1}^{f}\right) N_{3} d x_{k} \\
E\left[f\left(x_{k}\right) f^{T}\left(x_{k}\right)\right] \\
=\int f\left(x_{k}\right) f^{T}\left(x_{k}\right) N_{3} d x_{k} \\
E\left[\tilde{f}\left(x_{k \mid k-1}^{f}\right) \tilde{f}^{T}\left(X_{k \mid k-1}\right)\right] \\
=\int \tilde{f}\left(x_{k \mid k-1}^{f}\right) \tilde{f}^{T}\left(X_{k \mid k-1}\right) N_{4}^{k} d\left[\begin{array}{l}
x_{k} \\
X_{k}
\end{array}\right]
\end{gathered}
$$

The other expected terms are the same as the linear space of equation (147), so the probability density functions are all $N_{4}^{k}$. The integral formula for finding the expectation is similar and will not be repeated here.

$$
S_{k}^{W V}=\left[S_{k}^{W V^{1}}, \cdots, S_{k}^{W V^{N}}\right]^{T}
$$

where, $N_{3}=N\left(x_{k} ; x_{k \mid k-1}^{f}, P_{k \mid k-1}^{f}\right)$

$$
N_{4}^{k}=N\left(\left[\begin{array}{l}
x_{k} \\
X_{k}
\end{array}\right] ;\left[\begin{array}{c}
x_{k \mid k-1}^{f} \\
X_{k \mid k-1}
\end{array}\right],\left[\begin{array}{cc}
P_{k \mid k-1}^{f} & P_{k \mid k-1}^{f X} \\
\left(P_{k \mid k-1}^{f X}\right)^{T} & \Xi_{k \mid k-1}
\end{array}\right]\right) 。
$$

Based on the minimum mean square error criterion, $L_{k+1}$ is obtained as follows. Let

$$
\begin{aligned}
& \frac{\partial \operatorname{tr}\left(P_{k+1 \mid k}^{f}\right)}{\partial L_{k+1}}=0 \\
\Rightarrow & \mathrm{H}+\mathrm{M}=0
\end{aligned}
$$

where, $H$ and $M$ represent the first-order term and constant term after derivation, respectively. For the sake of clarity, the derivation process of $P_{k+1 \mid k}^{f}$ is divided into three parts, including the derivation of the $\Lambda$ part, the derivation of the $\Lambda^{T}$, and the derivation of the rest, which correspond to the first-order term and the constant term as
$\mathrm{H}_{1}, \mathrm{H}_{2}, \mathrm{H}_{3}, \mathrm{M}_{1}, \mathrm{M}_{2}, \mathrm{M}_{3}$. The details are as follows

$$
\begin{aligned}
\mathrm{H}_{1}= & -L_{k+1} e A-L_{k+1}(e A)^{T} \\
& -L_{k+1} B e-L_{k+1}(B e)^{T} \\
& -L_{k+1}\left(2 e Q_{k}^{W} e^{T}-e D-(e D)^{T}\right)
\end{aligned}
$$

Considering

$\frac{\partial \operatorname{tr}\left(\bar{A} \bar{B} \bar{A}^{T}\right)}{\partial \bar{A}}=\bar{A} \bar{B}+\bar{A} \bar{B}^{T}=\frac{\partial \operatorname{tr}\left(\bar{A} \bar{B}^{T} \bar{A}^{T}\right)}{\partial \bar{A}}$, one can obtain

$$
\mathrm{H}_{2}=\mathrm{H}_{1}
$$

$\mathrm{H}_{3}=2 L_{k+1} e C e^{T}+2 L_{k+1} e Q_{k}^{W} e^{T}+2 L_{k+1} \Xi_{k+1 k}$

where,

$$
\begin{gathered}
\mathrm{A}=\left\{\begin{array}{l}
\Phi_{k} E\left[\tilde{f}\left(x_{k \mid k-1}^{f}\right) \tilde{f}^{T}\left(X_{k \mid k-1}\right)\right] \Psi_{k}^{T} \\
-\Phi_{k} E\left[\tilde{f}\left(x_{k \mid k-1}^{f}\right) \tilde{h}^{T}\left(X_{k \mid k-1}\right)\right]\left(K_{k+1, k \mid k-1}^{x y} H_{k}\right)^{T}
\end{array}\right\} \\
\mathrm{B}=\left\{\begin{array}{l}
\Omega_{k}^{\tilde{\Phi} \tilde{\Psi}} E\left[f\left(x_{k}\right)\left(e f\left(x_{k}\right)\right)^{T}\right] \\
-\Omega_{k}^{\tilde{\Phi} \tilde{H}} E\left[f\left(x_{k}\right) h^{T}\left(x_{k}\right)\right]\left(K_{k+1, k \mid k-1}^{x y}\right)^{T}
\end{array}\right\} \\
C=\left\{\begin{array}{l}
\Phi_{k} E\left[\tilde{f}\left(x_{k \mid k-1}^{f}\right) \tilde{f}^{T}\left(x_{k \mid k-1}^{f}\right)\right] \Phi_{k}^{T} \\
+\Omega_{k}^{\tilde{\Phi} \tilde{\Phi}} E\left[f\left(x_{k}\right) f^{T}\left(x_{k}\right)\right]
\end{array}\right\} \\
D=S_{k}^{W V}\left(K_{k+1, k \mid k-1}^{x y}\right)^{T}
\end{gathered}
$$

Then,

$$
\left.\begin{array}{rl}
\mathrm{H}= & \mathrm{H}_{1}+\mathrm{H}_{2}+\mathrm{H}_{3} \\
= & 2 \mathrm{H}_{1}+\mathrm{H}_{3} \\
= & \left.\left(\begin{array}{l}
-L_{k+1} e A-L_{k+1}(e A)^{T} \\
-L_{k+1} B e-L_{k+1}(B e)^{T} \\
-L_{k+1}\left(2 e Q_{k}^{W W} e^{T}-e D-(e D)^{T}\right.
\end{array}\right)\right) \\
& +2 L_{k+1} e C e^{T}+2 L_{k+1} e Q_{k}^{W W} e^{T}+2 L_{k+1} \Xi_{k+1 \mid k} \\
= & -2 L_{k+1}\left(\begin{array}{l}
e A+(e A)^{T}+B e \\
+(B e)^{T}-e C e^{T}-\Xi_{k+1 \mid k} \\
+\left(e Q_{k}^{W W} e^{T}-e D-(e D)^{T}\right.
\end{array}\right)
\end{array}\right)
$$




$$
\begin{gathered}
M_{1}=A+B+Q_{k}^{W W} e^{T}-D \\
\text { Considering } \frac{\partial \operatorname{tr}\left(\bar{B} \bar{A}^{T}\right)}{\partial \bar{A}}=\frac{\partial \operatorname{tr}\left(\bar{A} \bar{B}^{T}\right)}{\partial \bar{A}}=\bar{B} \text {, then } \\
M_{2}=M_{1} \\
M_{3}=-2\left\{C+Q_{k}^{W}\right\} e^{T}
\end{gathered}
$$

and

$$
\begin{aligned}
M & =M_{1}+M_{2}+M_{3} \\
& =2\left(A+B+Q_{k}^{W} e^{T}-D\right)-2\left\{C+Q_{k}^{W}\right\} e^{T} \\
& =2\left(A+B-C e^{T}-D-Q_{k}^{W} e^{T}\right)
\end{aligned}
$$

Therefore,

$$
\begin{aligned}
& \mathrm{H}+\mathrm{M}=0 \\
& \Rightarrow L_{k+1}\left(\begin{array}{l}
e A+(e A)^{T}+B e+(B e)^{T} \\
+\left(e Q_{k}^{W W} e^{T}-e D-(e D)^{T}\right. \\
-e C e^{T}-\Xi_{k+1 k}
\end{array}\right) \\
& =\left(A+B+Q_{k}^{W W} e^{T}-D\right) \\
& -2\left\{\begin{array}{l}
\left.C+Q_{k}^{W W}\right\} e^{T} \\
\Rightarrow
\end{array} L_{k+1}=\left(\begin{array}{l}
\left(A+B+Q_{k}^{W W} e^{T}-D\right) \\
-2\left\{C+Q_{k}^{W W}\right\} e^{T}
\end{array}\right)\right. \\
& \times\left(\begin{array}{l}
e A+(e A)^{T}+B e+(B e)^{T} \\
+\left(e Q_{k}^{W W} e^{T}-e D-(e D)^{T}\right.
\end{array}\right) \\
& -e C e^{T}-\Xi_{k+1 k}
\end{aligned}
$$

Take $L_{k+1}$ and $A, B$ into (34) to get $\Lambda$. Take $L_{k+1}$ and $\Lambda$ into (33) to get a one-step prediction covariance, and take $L_{k+1}$ into (26) to get a one-step prediction mean.

\section{Appendix 2}

The proof of Theorem 2:

Proof:

$$
\begin{aligned}
\tilde{x}_{k+1 \mid k+1}^{f} & =x_{k+1}-x_{k+1 \mid k+1}^{f} \\
& =x_{k+1}-\left(I_{n}-L_{k+1} e\right) x_{k+1 \mid k}^{f}-L_{k+1} \hat{X}_{k+1 \mid k+1} \\
& =\left(I_{n}-L_{k+1} e\right) \tilde{x}_{k+1 \mid k}^{f}+L_{k+1} \tilde{X}_{k+1 \mid k+1}
\end{aligned}
$$

The local estimation error is rewritten as

$$
\begin{aligned}
& x_{k+1}-x_{k+1 \mid k+1}^{i}=x_{k+1}-x_{k+1 \mid k}^{i}-K_{k+1 k}^{x^{i} y^{i}} e_{k+1}^{i} \\
& =\tilde{x}_{k+1 \mid k}^{i}-K_{k+1 \mid k}^{x^{i} y^{i}}\left(\begin{array}{l}
H_{k+1}^{i} \tilde{h}^{i}\left(x_{k+1 \mid k}^{i}\right) \\
+\tilde{H}_{k+1}^{i} h^{i}\left(x_{k+1}\right)+V_{k+1}^{i}
\end{array}\right) \\
& =\left(\tilde{x}_{k+1 \mid k}^{i}-K_{k+1 \mid k}^{x^{i} y^{i}} H_{k+1}^{i} \tilde{h}^{i}\left(x_{k+1 \mid k}^{i}\right)\right) \\
& -K_{k+1 k}^{x^{i} y^{i}} \tilde{H}_{k+1}^{i} h^{i}\left(x_{k+1}\right)-K_{k+1 k}^{x^{i} y^{i}} V_{k+1}^{i}
\end{aligned}
$$

Sorting out local estimation errors yields

$$
\begin{gathered}
\tilde{X}_{k+1 \mid k+1}=\left(X_{k+1 \mid k}-K_{k+1 \mid k}^{x y} H_{k+1} \tilde{h}\left(X_{k+1 \mid k}\right)\right) \\
-K_{k+1 \mid k}^{x y} \tilde{H}_{k+1} h\left(x_{k+1}\right)-K_{k+1 \mid k}^{x y} V_{k+1}
\end{gathered}
$$

Similar to the definition of $K_{k+1, k \mid k-1}^{x y}$, we have $K_{k+1 \mid k}^{x y}=\operatorname{diag}\left(K_{k+1 \mid k}^{x^{1} y^{1}}, \cdots, K_{k+1 \mid k}^{x^{N} y^{N}}\right)$

The error covariance matrix is

$$
\begin{aligned}
P_{k+1 \mid k+1}^{f} & =E\left[\tilde{x}_{k+1 \mid k+1}^{f}\left(\tilde{x}_{k+1 \mid k+1}^{f}\right)^{T} \mid Y_{k+1}\right] \\
& =E\left[\left(\left(I_{n}-L_{k+1} e\right) \tilde{x}_{k+1 \mid k}^{f}+L_{k+1} \tilde{X}_{k+1 \mid k+1}\right)(\bullet)^{T}\right] \\
& =\left(I_{n}-L_{k+1} e\right) P_{k+1 k}^{f}\left(I_{n}-L_{k+1} e\right)^{T} \\
& +\left(I_{n}-L_{k+1} e\right) P_{k+1 \mid k, k+1}^{x^{f} X} L_{k+1}^{T}(\bullet)^{T} \\
& +L_{k+1} \Xi_{k+1 \mid k+1} L_{k+1}^{T}
\end{aligned}
$$

Based on the minimum mean square error criterion, we have

$$
\begin{aligned}
\frac{\partial \operatorname{tr} P_{k+1 \mid k+1}^{f}}{\partial L_{k+1}}= & -2 P_{k+1 \mid k}^{f} e^{T}+2 L_{k+1} e P_{k+1 \mid k}^{f} e^{T} \\
& +\left(P_{k+1 \mid k, k+1}^{x^{f} X}-2 L_{k+1} e P_{k+1 \mid k, k+1}^{x^{f} X}\right) \\
& +\left(P_{k+1 \mid k, k+1}^{x^{f} X}-2 L_{k+1}\left(P_{k+1 \mid k, k+1}^{x^{f} X}\right)^{T} e^{T}\right) \\
& +2 L_{k+1} \Xi_{k+1 \mid k+1} \\
= & 0
\end{aligned}
$$

Then, 


$$
\begin{aligned}
& L_{k+1}\left(e P_{k+1 \mid k}^{f} e^{T}-e P_{k+1 \mid k, k+1}^{x^{f} X}-\left(P_{k+1 \mid k, k+1}^{x^{f} X}\right)^{T} e^{T}+\Xi_{k+1 \mid k+1}\right) \\
& =P_{k+1 \mid k}^{f} e^{T}-P_{k+1 \mid k, k+1}^{x^{f} X} \\
& \Rightarrow L_{k+1}=\left(P_{k+1 \mid k}^{f} e^{T}-P_{k+1 \mid k, k+1}^{x^{f} X}\right) \\
& \left(e P_{k+1 \mid k}^{f} e^{T}-e P_{k+1 \mid k, k+1}^{x^{f} X}-\left(P_{k+1 \mid k, k+1}^{x^{f} X}\right)^{T} e^{T}+\Xi_{k+1 \mid k+1}\right)^{-1}
\end{aligned}
$$

where,

$$
\begin{aligned}
& P_{k+1 \mid k, k+1}^{x^{f} X}=E\left[\tilde{x}_{k+1 \mid k}^{f} \tilde{X}_{k+1 \mid k+1}^{T}\right]
\end{aligned}
$$

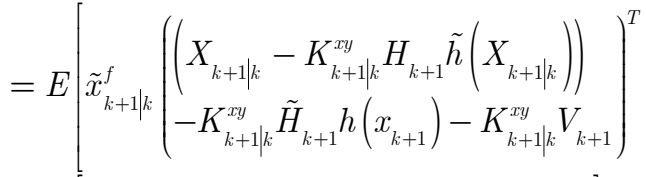

$$
\begin{aligned}
& =E\left[\tilde{x}_{k+1 \mid k}^{f}\left(X_{k+1 \mid k}-K_{k+1 \mid k}^{x y} H_{k+1} \tilde{h}\left(X_{k+1 \mid k}\right)\right)^{T}\right. \\
& =P_{k+1 \mid k}^{x^{f} X}-\int \tilde{x}_{k+1 \mid k}^{f} \tilde{h}^{T}\left(X_{k+1 k k}\right) N_{4}^{k+1} d\left[\begin{array}{c}
x_{k+1} \\
X_{k+1}
\end{array}\right]\left(K_{k+1 \mid k}^{x y} H_{k+1}\right)^{T}
\end{aligned}
$$

$$
\begin{aligned}
& \quad P_{k+1 \mid k}^{x^{f} X}=\left[P_{k+1 \mid k}^{x^{f} x^{1}}, \cdots, P_{k+1 \mid k}^{x^{f} x^{N}}\right]^{T}, \quad \text { and let } \\
& \text { where, } \\
& I_{n}-L_{k+1} e=\boldsymbol{\Psi} \text {, than }
\end{aligned}
$$

$$
\begin{aligned}
& P_{k+1 \mid k}^{x^{f} x^{i}}=\boldsymbol{\Psi} \Phi_{k} \int \tilde{f}\left(x_{k \mid k-1}^{f}\right) \tilde{f}\left(x_{k \mid k-1}^{i}\right) N_{5} d\left[\begin{array}{c}
x_{k} \\
x_{k}
\end{array}\right] \Phi_{k}^{T} \\
& -\boldsymbol{\Psi} \Phi_{k} \int \tilde{f}\left(x_{k \mid k-1}^{f}\right)\left(\tilde{h}^{i}\left(x_{k \mid k-1}^{i}\right)\right)^{T} N_{5} d\left[\begin{array}{l}
x_{k} \\
x_{k}
\end{array}\right] \times \\
& \left(K_{k+1, k \mid k-1}^{x^{i} y^{i}} H_{k}^{i}\right)^{T}+\boldsymbol{\Psi} \tilde{\Phi}_{k} \int f\left(x_{k}\right) f^{T}\left(x_{k}\right) N_{5} d\left[\begin{array}{l}
x_{k} \\
x_{k}
\end{array}\right]^{T} \\
& -\boldsymbol{\Psi} \tilde{\Phi}_{k} \int f\left(x_{k}\right)\left(h^{i}\left(x_{k}\right)\right)^{T} N_{5} d\left[\begin{array}{l}
x_{k} \\
x_{k}
\end{array}\right]\left(K_{k+1, k \mid k-1}^{x^{i} y^{i}} \tilde{H}_{k}^{i}\right)^{T} \\
& +\boldsymbol{\Psi}\left(Q_{k}^{W}-S_{k}^{W V^{i}}\left(K_{k+1, k \mid k-1}^{x^{i} y^{i}}\right)^{T}\right) \\
& +L_{k+1} P_{k+1 \mid k}^{X x^{i}}
\end{aligned}
$$

where, $N_{5}=N\left(\left[\begin{array}{c}x_{k} \\ x_{k}\end{array}\right] ;\left[\begin{array}{c}x_{k \mid k-1}^{f} \\ x_{k \mid k-1}^{i}\end{array}\right],\left[\begin{array}{cc}P_{k \mid k-1}^{f} & P_{k \mid k-1}^{x^{f} x^{i}} \\ \left(P_{k \mid k-1}^{x^{f} x^{i}}\right)^{T} & P_{k \mid k-1}^{x^{i} x^{i}}\end{array}\right]\right)$.

From the definition of $\tilde{X}_{k+1 \mid k}$, $P_{k+1 \mid k}^{X x^{i}}=\left[P_{k+1 \mid k}^{x^{1} x^{i}}, \cdots, P_{k+1 \mid k}^{x^{N} x^{i}}\right]^{T}$. Obviously the information in $P_{k+1 \mid k}^{X x^{i}}$ has been calculated in the one-step prediction process, so we can get the formula (169). Taking (169) into (168) yields $L_{k+1}$. Taking (168) and $L_{k+1}$ into (166) yields corrected covariance. Taking $L_{k+1}$ into (34) yields corrected estimation.

Received September 13, 2021

Accepted November 30, 2021 